Article

\title{
Precise Orbit Determination of BDS-2 and BDS-3 Using SLR
}

\author{
Honglei Yang, Tianhe $X u$ * , Wenfeng Nie, Fan Gao and Meiqian Guan \\ Institute of Space Science, Shandong University, Weihai 264209, China; hozzyee@mail.sdu.edu.cn (H.Y.); \\ wenfengnie@sdu.edu.cn (W.N.); gaofan@sdu.edu.cn (F.G.); 201820659@mail.sdu.edu.cn (M.G.) \\ * Correspondence: thxu@sdu.edu.cn; Tel.: +86-631-5622731
}

Received: 19 October 2019; Accepted: 19 November 2019; Published: 21 November 2019

\begin{abstract}
The BeiDou Navigation Satellite System (BDS) of China is currently in the hybrid-use period of BDS-2 and BDS-3 satellites. All of them are equipped with Laser Retroreflect Arrays (LRAs) for Satellite Laser Ranging (SLR), which can directly obtain an independent, sub-centimetre level of distance measurement. The main purpose of this contribution is to use the solely SLR Normal Points (NPs) data to determinate the precise orbit of BDS-2 and BDS-3 satellites, including one Geostationary Earth Orbit (GEO), three Inclined Geo-Synchronous Orbits (ISGO), and one Medium Earth Orbit (MEO) of BDS-2 satellites, as well as four MEO of BDS-3 satellites, from 1 January to 30 June 2019. The microwave-based orbit from Wuhan University (WUM) are firstly validated to mark and eliminate the bad SLR observations in our preprocessing stage. Then, the 3-, 5-, 7-, and 9-day arc solutions are performed to investigate the impact of the different orbital arc lengths on the quality of SLR-derived orbits and test the optimal solution of the multi-day arc. Moreover, the dependency of SLR-only orbit determination accuracy on the number of SLR observations and the number of SLR sites are discussed to explore the orbit determination quality of the 3-,5-, 7-, and 9-day arc solutions. The results indicate that (1) during the half-year time span of 2019, the overall Root Mean Square (RMS) of SLR validation residuals derived from WUM is $19.0 \mathrm{~cm}$ for BDS-2 GEO C01, 5.2-7.3 cm for three BDS-2 IGSO, $3.4 \mathrm{~cm}$ for BDS-2 MEO C11, and 4.4-5.7 cm for four BDS-3 MEO satellites respectively. (2) The 9-day arc solutions present the best orbit accuracy in our multi-day SLR-only orbit determination for BDS IGSO and MEO satellites. The 9-day overlaps median RMS of BDS MEO in RTN directions are evaluated at 3.6-5.7, 12.4-21.6, and 15.6-23.9 cm respectively, as well as 5.7-9.6, 15.0-36.8, and 16.5-35.2 cm for the comparison with WUM precise orbits, while these values of BDS IGSO are larger by a factor of about 3-10 than BDS MEO orbits in their corresponding RTN directions. Furthermore, the optimal average 3D-RMS of 9-day overlaps is 0.49 and $1.89 \mathrm{~m}$ for BDS MEO and IGSO respectively, as well as 0.55 and $1.85 \mathrm{~m}$ in comparison with WUM orbits. Owing to its extremely rare SLR observations, the SLR-only orbit determination accuracy of BDS-2 GEO satellite can only reach a level of 10 metres or worse. (3) To obtain a stable and reliable SLR-only precise orbit, the 7-day to 9-day arc solutions are necessary to provide a sufficient SLR observation quantity and geometry, with more than 50-80 available SLR observations at 5-6 SLR sites that are evenly distributed, both in the Northern and Southern Hemispheres.
\end{abstract}

Keywords: BDS-2; BDS-3; SLR; Precise Orbit Determination; Precise Orbit Validation

\section{Introduction}

Currently, the BeiDou Navigation Satellite System (BDS) of China is in the period when BDS-2 and BDS-3 satellites are used together, and both generations of BDS continue to reinforce and complete their constellation. According to official reports, China has successfully launched its 45th BDS satellite, which is the fourth backup satellite of BDS-2, on 17 May 2019. Also, the 47th and 48th BDS 
satellites, as the 22th and 23th satellites of BDS-3, were successfully launched on 23 September 2019 (http://www.beidou.gov.cn/). In the three generations of BDS, BDS-1 has been completely retired. BDS-2 continues to operate and serve the world with a focus on the Asia-Pacific area at a constellation of 14 satellites, including five Geostationary Orbit (GEO) satellites, five Inclined Geosynchronous Orbit (IGSO) satellites, and four Medium Earth Orbit (MEO) satellites. So far, BDS-3 is gradually replenishing satellites to achieve full constellation operation with three GEO, three IGSO, and 24 MEO satellites in 2020 [1,2]. All the BDS satellites are equipped with satellite Laser Retroreflect Arrays (LRAs) for Satellite Laser Ranging (SLR). The SLR, as a unique geodetic survey technique without ambiguity, can directly obtain an independent, sub-centimetre level of satellite-station distance measurement.

Since the rise of Global Navigation Satellite System (GNSS) technology in the 1990s, two American Global Positioning System (GPS) satellites with LRAs—namely GPS35 and GPS36-had been tracked by SLR. Their basic characteristics and performance, such as the principles, the tracking properties, and their LRAs, were described in Degnan and Pavlis [3]. The encouraging result was that for the two special sets of SLR-only determination orbits of GPS35 with 14-day arcs, the Root Mean Square (RMS) of the difference in the orbital overlaps was $3.2,37.0$, and $10.9 \mathrm{~cm}$, while the mean residual offset is 5.1, 21.8 , and $-19.0 \mathrm{~cm}$ in terms of radial, cross-track, and along-track directions respectively. Compared with the orbits provided by the International GNSS Service (IGS), the orbit difference was about $10 \mathrm{~cm}$ in the radial direction, and 0.5-1.0 $\mathrm{m}$ in the cross-track and along-track directions [4]. In the technical report of Schutz [5], the RMS was about 24-31, 94-125, and 49-71 cm in Radial-Transverse-Normal (RTN) directions respectively, based on the comparison of GPS-35 L-band orbits with those of SLR, where the L-band orbits came from five different Analysis Centers (ACs). In addition, the optimal RMS can reach $8 \mathrm{~cm}$ after Helmert transformation in the middle 1-day of a 9-day arc, comparing the difference between the SLR-only and the IGS orbits of GPS35 [6].

With the International GLONASS (GLObal NAvigation Satellite System) Experiment 98 (IGEX-98) campaign initialized, the GLONASS orbits were computed and compared using SLR and microwave-based data. The GLONASS satellites had a slightly more accurate SLR orbit than GPS35, with the RMS of 10, 40, and $45 \mathrm{~cm}$ in RTN directions respectively, even in the case of the mismodelled Solar Radiation Pressure (SRP) force assignment [7]. Comparing the differences between the SLR and microwave orbits for GPS and GLONASS orbits, the RMS was about $10 \mathrm{~cm}$ in the radial and about $50 \mathrm{~cm}$ both in the along-track and across-track directions, as reported in Appleby \& Otsubo (2000), where the Helmert transformation and SLR system-dependent range bias were also discussed to explore the sources of systematic error.

In terms of GLOVE-A, the first Galileo In-Orbit Validation Element (GLOVE) satellites, the RMS was 8,45 , and $37 \mathrm{~cm}$ in RTN directions for the orbit overlap misfit between a 10-day and a 30-day arc. The temporal evolution of mean metric elements of GLOVE-A, GPS-35, and GPS-36 satellites were studied to investigate the orbital dynamic characteristics [8]. A set of orbit difference between 3-day microwave-only and 7-day SLR-only solution was obtained by 9.3, 51.0, and $39.6 \mathrm{~cm}$ in RTN directions, as well as $65.2 \mathrm{~cm}$ for the corresponding 3D-RMS [9]. Meanwhile, the SLR-only orbit overlap of 9-day arcs for the SLR-only GLOVE-A satellite were about $10 \mathrm{~cm}, 0.5 \mathrm{~m}$, and $1 \mathrm{~m}$ [10]; the same results were obtained in Flohrer [11].

For BDS, the 3D-RMS of BeiDou-G1, -I3, -I5, and -M3 were on the level of one metre to a few metres, while the radial accuracy was on the level of decimetres [12]. In Zhao et al. [13], the 7-day arcs of SLR-only orbits for GPS, GLONASS, Galileo, and BDS were implemented, and the orbit accuracy were evaluated and compared, based on the different satellite types, as well as on different satellite constellations. For MEO, the radial accuracy was around 4-10 cm while the 3D-RMS are tens of $\mathrm{cm}$, either for orbit overlaps or for comparisons with microwave-based orbits.

Overall, most previous studies show that for a selected arc, the SLR-derived orbit for the GNSS satellites is not universal. It is not quite clear what accuracy can be achieved under different conditions. The multi-GNSS orbit of long-term time series-using solely SLR observations-was determined by Bury et al. [14] and Bury et al. [15]. The contribution demonstrated that 60 SLR observations—as many 
SLR sites as possible-and optimal 5-day to 7-day arc length were necessary in order to provide a better geometry of observations and preclude deterioration of the along-track component caused by excessive arc length. However, the SLR-only orbit for BDS is not analysed in detail, especially for the BDS GEO satellites. Moreover, the SLR-only orbit determination for BDS-3 satellites, as a fresh constellation, has hardly been explored. Based on these, the SLR-only orbit for BDS-2 and BDS-3 satellites are determined from 1 January 2019 to 30 June 2019 in this contribution. The 3-, 5-, 7-, and 9-day arc solutions are calculated to test and determine the magnitude of accuracy of the SLR-only orbit of BDS-2 and BDS-3 satellites, including a total of nine BDS satellites, which cover all the three types of satellites: GEO, IGSO, and MEO. Among them, five satellites belong to BDS-2-namely C01, C08, C10, C11, and C13. The remaining four satellites belong to BDS-3, which are C20, C21, C29, and C30. The accuracy of SLR-only orbit determination is evaluated by the orbit overlaps and by the comparison with the microwave-based precise orbit of WUM. Besides, the dependency of SLR-only orbit determination accuracy on the number of SLR observations and on the number of SLR sites is explored in detail.

The paper is organized as follows. In Section 2, the methodology of SLR-only orbit determination and the corresponding accuracy assessment strategy are described. Then, Section 3 presents the time series of processed SLR data and the SLR validation residuals derived from WUM while the results of SLR-only orbit determination are shown in Section 4. Section 5 discusses the accuracy dependency of the SLR-only orbit determination on the number of SLR observations and on the number of SLR sites. Finally, we summarize and comment on the conclusions in the last section.

\section{Methodology}

\subsection{Orbit Determination Strategy}

The methodology of SLR-only orbit determination is consistent with that of Bury, Sośnica and Zajdel [15], while the strategy of configuration of some parameters is changed and applied to BDS GEO, as well as for BDS-3 satellites, in this contribution. As shown in Table 1, the microwave-based precise orbits and the corresponding Earth Rotation Parameter (ERP) derived from WUM (ftp: //cddis.gsfc.nasa.gov/gnss/products/mgex/) are used to estimate the a priori orbital parameters, as well as the 9-parameter Empirical CODE Orbit Model (ECOM) SRP model [16]. As for the other dynamic model parameters, the N-body perturbation of the Sun, the Moon, and the major planets (Jupiter, Venus, and Mars) are considered using JPL DE405 [17] while the Earth gravity field terms are limited to the degree and order of 12 of the EGM2008 model. In addition, the SLR site displacement models are consistent with the Earth Rotation and Reference System Service (IERS) conventions 2010 [18], including the solid Earth tide, the related pole tides, the ocean tidal loading, and the general relativistic effect. The SLR site coordinates are fixed to the a priori reference frame SLRF2014 (ftp://cddis.nasa.gov/slr/products/resource/), with the corresponding corrections of the site coordinate eccentricity, as presented in ftp://cddis.gsfc.nasa.gov/slr/slrocc/ecc_une.snx. Besides, the troposphere delay is corrected via the Mendes-Pavlis model [19]. For the estimated parameters, the station coordinates are strongly constrained to $10^{-5}$ metres in each direction, while the ERP are fixed to the corresponding a priori value provided by WUM. A range bias is estimated simultaneously with other parameters in each multi-day arc solution for all available SLR sites and each BDS satellite. The SLR range bias parameters are constrained to a priori sigma of $0.1 \mathrm{~m}$ with no constraints to the Keplerian and empirical orbit parameters. The 'ALL' for range biases in Table 1 means that a range bias is estimated for all available SLR site and each BDS satellite. Moreover, the offset of the Centre-of-Mass of LRAs for BDS-2 satellites is fixed the same as in Yang et al. [20]. The China Academy of Space Technology (CAST) and the Shanghai Engineering Center for Microsatellites (SECM), as the two manufacturers of BDS-3, calibrated the Centre-of-Mass offset of LRAs related to the centre of mass of the satellite body for BDS-3 satellites respectively, as shown in Table 2. 
Table 1. The configuration of parameters of Satellite Laser Ranging (SLR)-only orbit determination.

\begin{tabular}{cc}
\hline Dynamic Models & Description for BDS SLR \\
\hline Earth Gravity Field & EGM2008 \\
N-body Perturbation & JPL DE405 \\
Solar Radiation Pressure & ECOM \\
Tides and Relativistic & IERS2010 \\
\hline Measurement Models & \\
\hline Basic Observations & SLR NPs \\
A priori Orbital Parameters & Precise orbit of WUM \\
A priori Station Coordinates & SLRF2014 \\
A Priori Troposphere Model & Mendes-Pavlis model \\
Centre-of-Mass Correction of LRAs & ILRS, CAST and SECM \\
\hline Estimated Parameters & Strong constrained \\
\hline Station Coordinates & None \\
Orbital Parameters & Fixed ERP of WUM \\
Pseudo-Random Pulse & None \\
ERP & All \\
Geocentre Coordinates & \\
Range Bias & Orbital Element and 9 ECP SR
\end{tabular}

Table 2. The Centre-of-Mass Correction of LRAs for BDS-3 satellites.

\begin{tabular}{ccc}
\hline & $\mathrm{C20} / \mathrm{C21}(\mathrm{m})$ & $\mathbf{C 2 9} / \mathrm{C30}(\mathrm{m})$ \\
\hline $\mathbf{X}$ & 0.6120 & 0.6646 \\
$\mathbf{Y}$ & -0.072 & 0.4249 \\
$\mathbf{Z}$ & 1.2230 & 0.6427 \\
\hline
\end{tabular}

Figure 1 shows the multi-day arcs solution strategy in this contribution. The whole procedure is implemented in the batch processing mode by moving time window. The procedure is realized through our modification of the Bernese GNSS Software 5.2 [21]. At first, the SLR Normal Points (NPs) data is preprocessed by comparing the station-satellite distance between SLR and the microwave-based priori orbit from WUM. For BDS IGSO and MEO, when the residuals of the SLR observations exceed $300 \mathrm{~mm}$, the observations are eliminated in our preprocessing stage, while for BDS GEO satellites, the corresponding threshold is 1,000 $\mathrm{mm}$. Then, the 1-day parameters are estimated and the corresponding normal equations (NEQs) are generated synchronously, based on the clean SLR observations. The multiple 1-day NEQs are combined into the multi-day NEQs in accordance with to the moving time window sequence. Subsequently, each set of stacked multi-day NEQs estimates a set of corresponding orbital parameters. This classic methodology was initially implemented by Beutler et al. [22], and it has been routinely applied to the GNSS products solution for CODE AC [23,24], as well as the SLR-only orbit determination for Bury, Sośnica and Zajdel [15]. Additionally, the equal weight scheme is adopted for all available SLR observations in both our SLR validation and SLR-only orbit determination strategies.

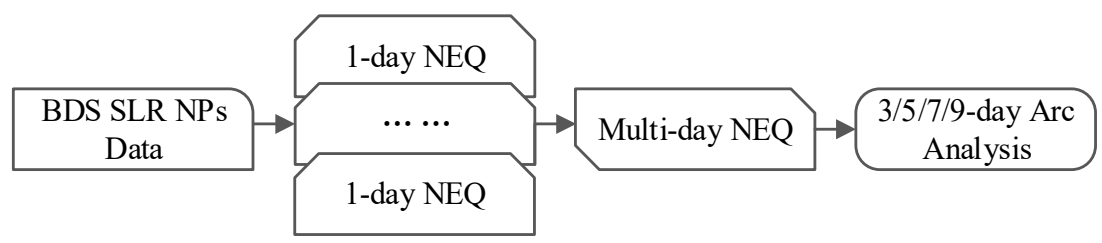

Figure 1. The multi-day arcs solution strategy. 


\subsection{Orbit Accuracy Assessment}

The difference of orbit overlap is obtained as an internal accuracy indicator by comparing the two adjacent multi-day arc solutions, where the middle day of the first multi-day arc is compared with the corresponding day of the second multi-day arc. Meanwhile, the external accuracy of the multi-day arc solutions is evaluated by comparing the difference with microwave-based precise orbit derived from WUM. The orbit differences are decomposed into RTN directions at 15-min intervals, and their corresponding daily RMS are calculated. Affected by the different quantities of the SLR observation and the types of BDS satellites, the accuracy of the SLR-only orbit may sometimes be unacceptable. To obtain the credible average RMS of long-term time series, the RMS of multi-day arc solutions are eliminated empirically and regarded as a failure in SLR-only orbit determination when the average RMS exceeds the threshold, which is 1,5 , and $5 \mathrm{~m}$ in $\mathrm{R}, \mathrm{T}$, and $\mathrm{N}$ directions for BDS MEO satellites, and 2, 10, and $10 \mathrm{~m}$ for BDS IGSO, as well as 10, 50, and $50 \mathrm{~m}$ for BDS GEO C01. Then the corresponding average RMS is calculated as the overall accuracy indicators. Additionally, to reflect the dispersion of the average RMSs, an index-which refers to the proportion of the RMS values that are under a quarter of the RMS threshold—is defined as $D$-value. Its calculation equation is as follows:

$$
D=\frac{M}{N} \times 100
$$

where $N$ refers to the number of success solutions and $M$ means the number of success solutions, the RMS of which is under the one-fourth RMS threshold.

\section{Processed Data and SLR Validation}

\subsection{SLR NPs Data of BDS Satellites}

All available SLR NPs observations for BDS-2 and BDS-3 satellites, provided by the Europe Data Centre (EDC) (ftp://edc.dgfi.tum.de/pub/slr/data/npt_crd/), have been collected and adopted from 1 January 2019 to 30 June 2019 in our processing, as shown in Figure 2. In the figure, the satellite with the superscript ${ }^{\prime * \prime}$ refers to the BDS-3 satellites. The BDS-2 MEO C11 owns the most observations among the nine BDS satellites, with the number of daily observations up to 50 , while the daily observations for GEO C01 are no more than 10. The SLR observation distribution pattern of IGSO C13 is no worse than that of the four BDS-3 satellites in this time span. It is noticed that almost no SLR observation is provided around 10 February 2019 for all BDS satellites. It happens to be during the Chinese New Year holiday, while the SLR site in Shanghai (SHA) is the only provider of the BDS satellites' orbit predictions for all SLR sites.

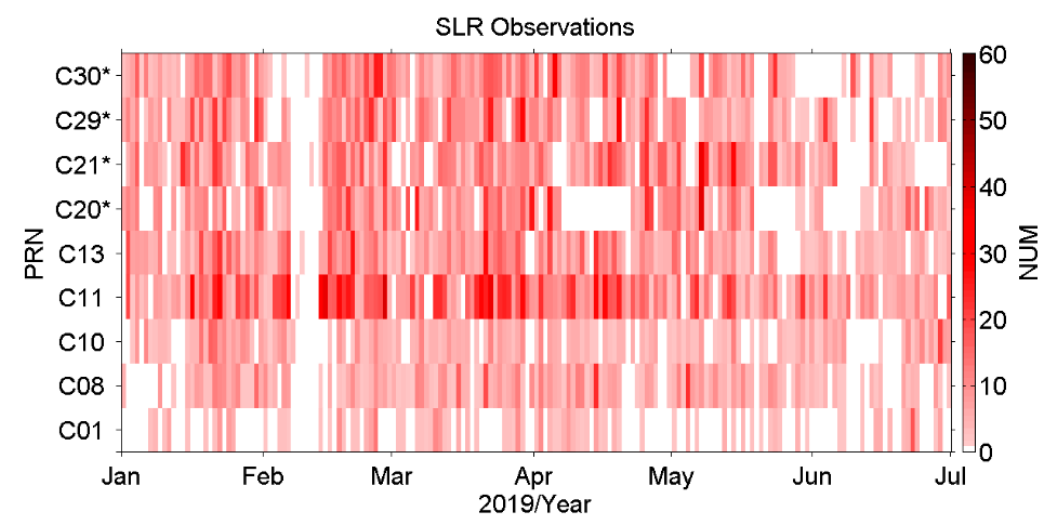

Figure 2. The daily SLR observations of BeiDou Navigation Satellite System (BDS) provided by Europe Data Centre (EDC) since 2019. 
Figure 3 shows the contribution of each SLR site to the daily SLR observations of all BDS satellites. In the figure, the SLR sites with the superscript ${ }^{* \prime}$ refer to the location of the site in the Southern Hemisphere. Currently, seven SLR sites are active in the Southern Hemisphere, as presented in the website https:/ilrs.cddis.eosdis.nasa.gov/network/stations/index.html. The Yarragadee (7090) site and Mount Stromlo (7825) site, located in the southwest and southeast of mainland Australia, in addition to the Changchun (7237) site from China, have made outstanding contributions to the SLR observations of BDS satellites. For the other sites, such as 7810 (Zimmerwald, Switzerland), 7839 (Graz, Austria), 7840 (Herstmonceux, United Kingdom), 7845 (Grasse, France), 7941 (Matera, Italy), and 8834 (Wettzell, Germany), their SLR observations are concentrated in the BDS MEO satellites.

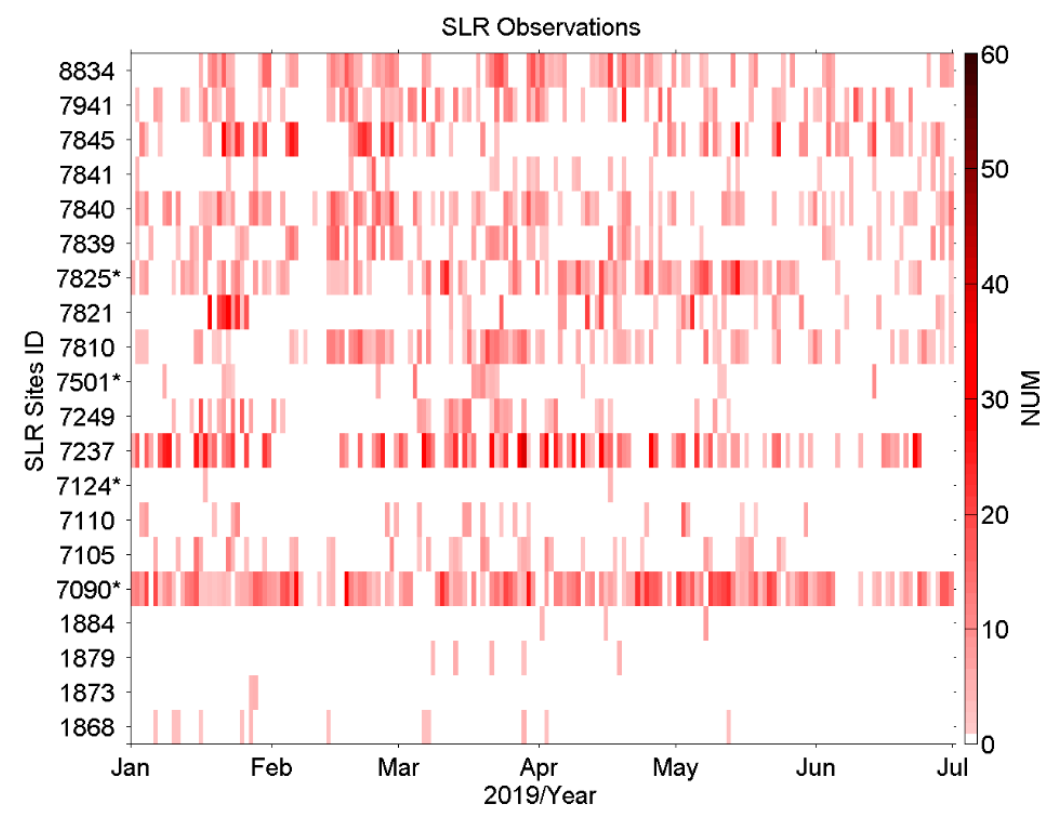

Figure 3. The contribution of each SLR site to the daily SLR observations of all BDS satellites.

\subsection{SLR Validation}

As a unique geodetic survey technology without ambiguity, the SLR technology has always been used to validate the GNSS microwave-based orbits externally and to investigate the deficiency of the error model in the microwave-based orbit determination [20,25-30]. In this contribution, the motivation of the SLR validation is to flag and eliminate the worse SLR observations, which can improve the precision and stability of SLR-only orbit determination.

In Table 3, the percentage of qualified SLR observations is over 95.5\% for all BDS satellites, with the number of available SLR observations of 237, 554-972, 1712, and 808-1037 for BDS-2 GEO C01, BDS-2 IGSO, BDS-2 MEO C11, and BDS-3 MEO satellites respectively. The RMS of SLR residuals is $19.0 \mathrm{~cm}$, and the mean offset is $-7.5 \mathrm{~cm}$ for the unique BDS GEO C01. As the only BDS-2 MEO satellites, the RMS of C11 is $3.4 \mathrm{~cm}$ with the mean offset of $0.9 \mathrm{~cm}$. The SLR residuals pattern are no significant variation for BDS MEO satellites in the selected period, including in the YM period. While the orbit accuracy becomes worse during eclipse period for the three IGSO satellites, especially in the YM period due to the inaccuracy attitude modelling. But they still have the credible RMS of $5.2-7.3 \mathrm{~cm}$, with the mean offset of $-4.2--1.8 \mathrm{~cm}$. Notably, the accuracy of the new microwave-based precise orbit derived from WUM has a significant improvement by almost $30 \mathrm{~cm}$ for GEO C01 comparing with the previous SLR validation results before March 2018 [20]. Moreover, the SLR residuals do not decrease significantly during the eclipse period for MEO C11, even during YM period. 
Table 3. The statistical information of the percentage of the available SLR observations, the number of the available SLR observations, and the Mean/RMS for SLR validation residuals (Unit: $\mathrm{cm}$ ).

\begin{tabular}{cccccccc}
\hline PRN & Perc. & Num. & Overall & Noneclip. & Eclip. & NYS & YM \\
\hline C01 & $99.6 \%$ & 237 & $-7.5 / 19.0$ & $-4.2 / 17.6$ & $-12.8 / 21.1$ & $-/-$ & $-/-$ \\
C08 & $96.6 \%$ & 593 & $-4.2 / 7.3$ & $-4.2 / 6.9$ & $-5.7 / 11.8$ & $-0.8 / 3.0$ & $-16.8 / 20.8$ \\
C10 & $95.5 \%$ & 554 & $-3.3 / 6.6$ & $-3.8 / 6 / 0$ & $-0.5 / 9.2$ & $-1.0 / 8.2$ & $0.1 / 10.1$ \\
C13 & $97.9 \%$ & 972 & $-1.8 / 5.2$ & $-1.6 / 5.1$ & $-3.2 / 6.0$ & $-1.7 / 4.6$ & $-4.8 / 7.3$ \\
C11 & $97.8 \%$ & 1712 & $0.9 / 3.4$ & $1.1 / 3.4$ & $0.4 / 3.2$ & $0.9 / 3.1$ & $-1.7 / 3.8$ \\
C20 & $98.1 \%$ & 808 & $0.3 / 4.6$ & $0.3 / 4.5$ & $0.2 / 5.0$ & $0.3 / 4.7$ & $0.2 / 5.7$ \\
C21 & $98.8 \%$ & 1037 & $0.0 / 4.4$ & $0.1 / 4.2$ & $-0.6 / 5.4$ & $-1.3 / 5.6$ & $0.9 / 5.0$ \\
C29 & $97.6 \%$ & 922 & $-0.4 / 5.7$ & $-0.5 / 5.9$ & $-0.1 / 4.8$ & $0.9 / 5.0$ & $-2.2 / 4.3$ \\
C30 & $99.1 \%$ & 889 & $-1.4 / 5.0$ & $-1.5 / 5.0$ & $-1.0 / 4.8$ & $-0.9 / 4.7$ & $-1.2 / 5.3$ \\
\hline
\end{tabular}

Similarly, the time series of SLR residual patterns do not have significant deterioration for BDS-3 during the eclipse period, as well as during the YM period, as shown in Figures 4 and 5. And their overall mean offset and RMS of BDS-3 MEO satellites is $-1.4-0.3 \mathrm{~cm}$ and $4.4-5.7 \mathrm{~cm}$ respectively. The excellent SLR validation results of the WUM's microwave-based orbits are attributed to the improved microwave-based orbit determination algorithm, especially the Yaw attitude modelling, and some systematic error compensation for BDS satellites, particularly for GEO C01 [31,32]. In addition, no orbit accuracy decreases for BDS-3 occurs in the eclipse period, especially in the YM period. An important factor is caused by the different attitude control modes between BDS-2 and BDS-3 satellites in the YM period. The empirical Normal Yaw Steering (NYS) mode of BDS-3 replaces the Orbit Fixed Yaw (OFY) mode of BDS-2 in this period. The experienced manoeuvring only occurs in noon- and midnight-point, as in an approximately range of $\beta \in\left[-3^{\circ}, 3^{\circ}\right]$, for BDS-3 satellites [31].

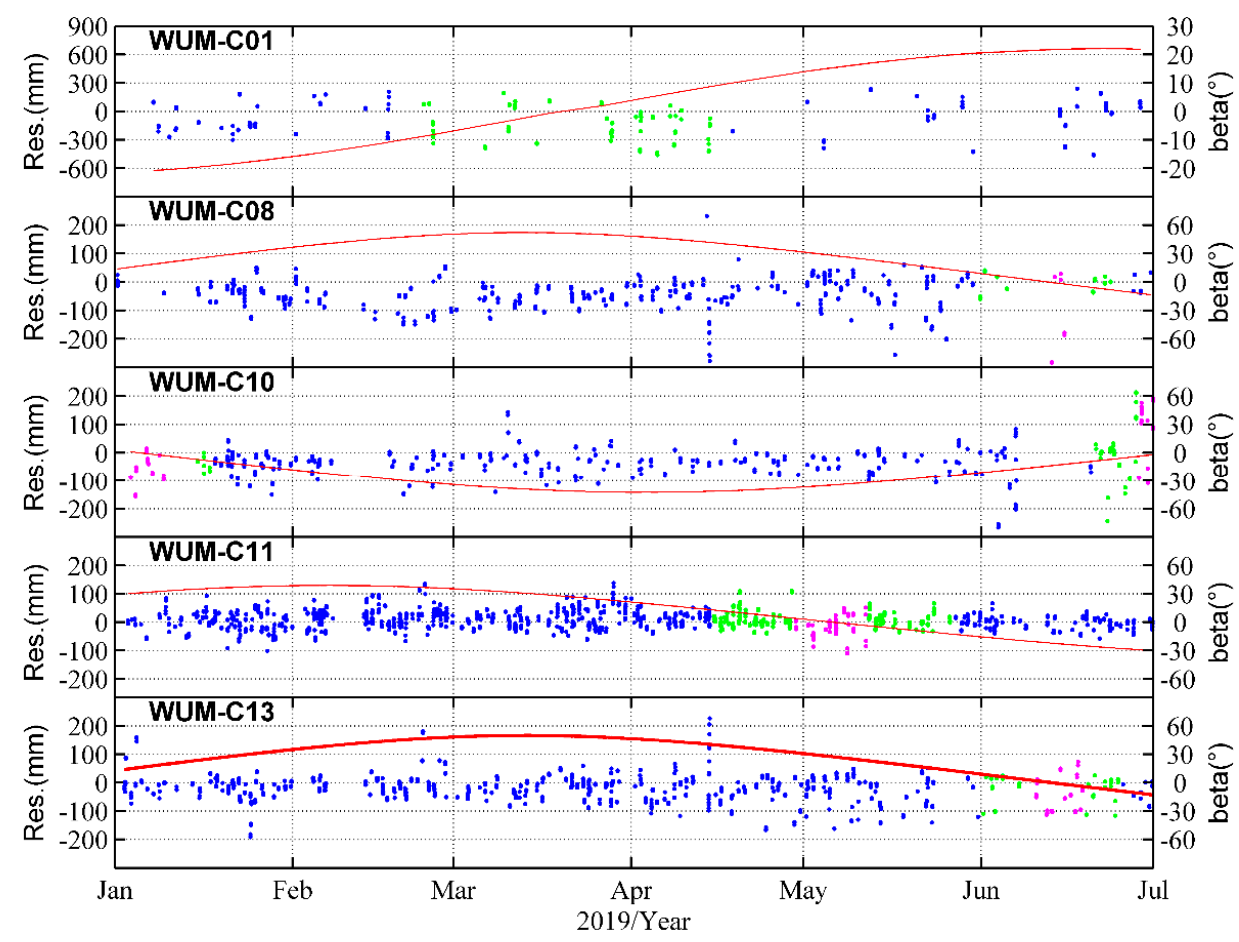

Figure 4. The SLR validation time series for BDS-2 satellites derived from WUM in the half-year time series since 2019. The blue scatters, green scatters, magenta scatters, and red line refer to the Sun elevation angle of $\beta$, the SLR residuals during the non-eclipse period, the Normal Yaw Steering (NYS) of the eclipse period, and the Yaw Manoeuver (YM) period respectively. The definitions of these concepts for BDS-2 satellites were summarized and described in Yang, Xu, Nie, Gao and Guan [20], and we apply exactly the same SLR validation strategy in this contribution. 


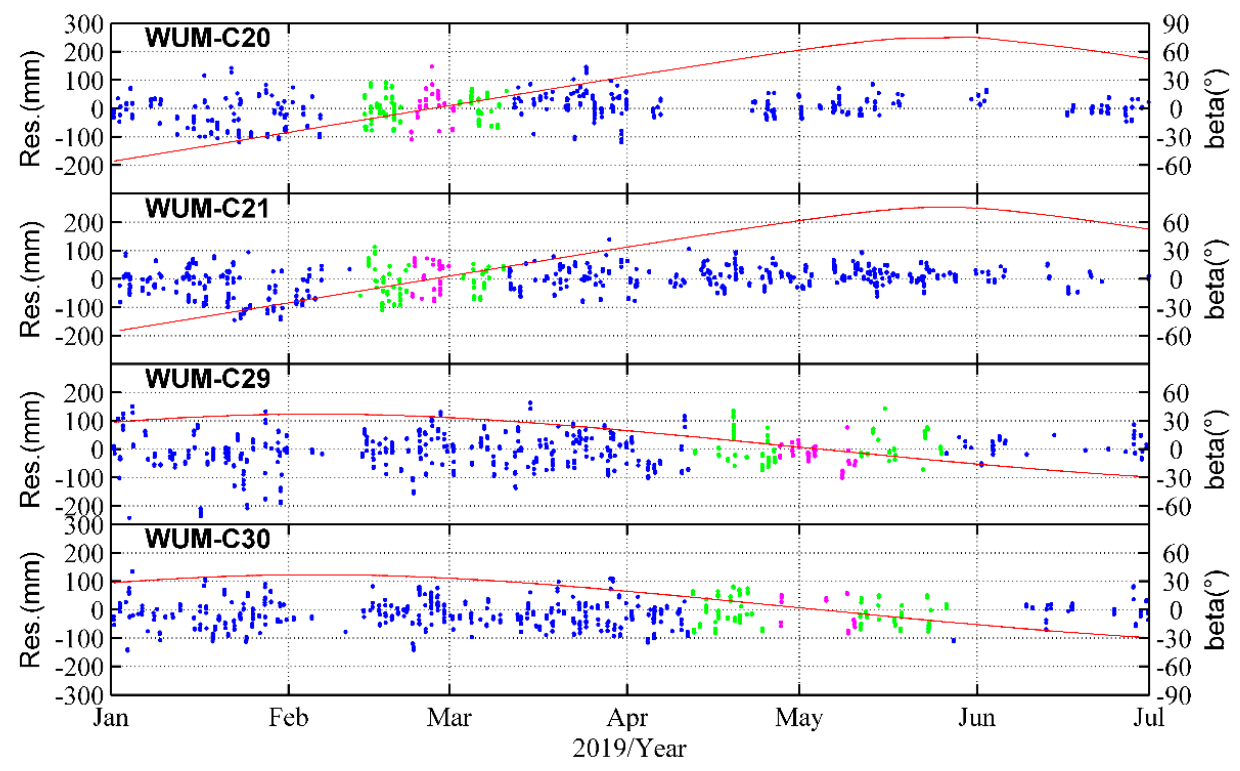

Figure 5. The SLR validation time series for BDS-3 satellites derived from WUM in the half-year time series since 2019. The definition of different colour dots and the red line are the same as in Figure 4.

The orbit accuracy of SLR validation is in at the decimetre level for GEO C01, while at the centimetre level for IGSO and MEO satellites. Therefore, we adopt different data preprocessing strategies and different statistical strategies for the SLR-only orbit determination of different BDS satellites, as described in Section 2.2. In addition, the percentage of qualified SLR observations is over 95.5\% for all BDS satellites, indicating that the preprocessing strategies are reasonable and reliable, especially for BDS-2 GEO C01 and BDS-3 satellites.

\section{Results}

The 3-, 5-, 7-, and 9-day arc solutions are displayed from different aspects to fully evaluate the accuracy and stability of SLR-only orbit determination for BDS satellites. A total of 173 RMS groups of SLR multi-day arc solutions are analysed from the half-year time series in 2019. To avoid the contamination of bad multi-day arc solutions, these bad RMS of multi-day arc solutions are regarded as failure solutions, based on the strategy described in Section 2.2. The success rate of multi-day solutions for all nine BDS satellites is presented in Section 4.1. Then Section 4.2 shows the $D$-value, the pattern of each average RMS value, and the Sun elevation angle of $|\beta|$, as well as the overall average RMS values of the orbit overlaps and comparison with WUM orbits in RTN directions. The overall average 3D-RMS is presented in Section 4.3. Section 4.4 analyses the median RMS of 9-day solutions.

\subsection{The Success Rate of Multi-day Solutions}

In Table 4, we find that the percentage of success solutions increases dramatically with the increase in the arc length, except for the comparison with WUM orbit of GEO C01. Although the success rate of C01 improves from $19.8 \%$ of the 3 -day solution to $37.2 \%$ of the 9 -day solution for the orbit overlaps, it decreases from $12.7 \%$ of the 3 -day solution to $8.1 \%$ of the 9 -day solution for the comparison with WUM orbit. Among the three BDS IGSO, the success rate of C13 in the 9-day success solution can reach $89.0 \%$ for the orbit overlaps and $85.5 \%$ for the comparison with WUM orbit. In particular, from the 3-day solution to the 9-day solution, the success rate of C11 increases from $53.2 \%$ to $100 \%$ for the comparison with WUM orbit, as well as $43.0 \%$ to $99.4 \%$ for the orbit overlaps. The success rate is also more than $80 \%$ for the orbit overlaps of BDS-3 MEO C21 and C29; however, it is only $58.7 \%$ for BDS-3 MEO C20, caused by the interruption of nearly a month of SLR observation data for C20, and a similar situation appears in C30 (as seen in Figure 2). 
Table 4. The success rate of multi-day solutions.

\begin{tabular}{ccccccccc}
\hline \multirow{2}{*}{ PRN } & \multicolumn{9}{c}{ Overlaps } & \multicolumn{4}{c}{ Vs WUM } \\
\cline { 2 - 8 } & 3-Day & 5-Day & 7-Day & 9-Day & 3-Day & 5-Day & 7-Day & 9-Day \\
\hline C01 & $19.8 \%$ & $22.1 \%$ & $29.1 \%$ & $37.2 \%$ & $12.7 \%$ & $8.7 \%$ & $5.8 \%$ & $8.1 \%$ \\
C08 & $9.3 \%$ & $22.1 \%$ & $52.3 \%$ & $62.2 \%$ & $9.8 \%$ & $22.5 \%$ & $45.1 \%$ & $55.5 \%$ \\
C10 & $7.0 \%$ & $11.0 \%$ & $27.3 \%$ & $45.3 \%$ & $5.8 \%$ & $9.8 \%$ & $30.1 \%$ & $46.2 \%$ \\
C13 & $12.2 \%$ & $44.2 \%$ & $75.6 \%$ & $89.0 \%$ & $15.6 \%$ & $50.3 \%$ & $76.3 \%$ & $85.5 \%$ \\
C11 & $43.0 \%$ & $77.9 \%$ & $93.0 \%$ & $99.4 \%$ & $53.2 \%$ & $82.1 \%$ & $95.4 \%$ & $100 \%$ \\
C20 & $11.6 \%$ & $43.0 \%$ & $54.7 \%$ & $58.7 \%$ & $15.0 \%$ & $41.6 \%$ & $50.9 \%$ & $56.1 \%$ \\
C21 & $27.9 \%$ & $60.5 \%$ & $76.2 \%$ & $80.8 \%$ & $36.4 \%$ & $57.8 \%$ & $70.5 \%$ & $71.7 \%$ \\
C29 & $23.8 \%$ & $52.9 \%$ & $75.6 \%$ & $82.0 \%$ & $28.9 \%$ & $54.3 \%$ & $73.4 \%$ & $76.3 \%$ \\
C30 & $20.9 \%$ & $51.7 \%$ & $65.1 \%$ & $72.1 \%$ & $22.5 \%$ & $45.7 \%$ & $59.5 \%$ & $63.6 \%$ \\
\hline
\end{tabular}

\subsection{The Orbit Overlaps and Comparison with WUM Orbits}

The results of 3-, 5-, 7-, and 9-day arc solutions are presented for all nine BDS satellites from Figures 6-14 while the red, blue, magenta, and green colours in the histogram and scatter chart correspond to the results of 3-, 5-, 7-, and 9-day solutions. In each figure, the top three sub-graphs show the SLR-only orbit overlap errors between two adjacent multi-day arc solutions in the R, T, and $\mathrm{N}$ directions while the bottom three sub-graphs display the difference between the SLR-only orbit and microwave-based precise orbit of WUM. Meanwhile, the left four columns represent the histogram of the number of success solutions, where the vertical axis refers to the RMS for orbital difference and the horizontal axis refers to the number of success solutions that are binned into 100 pieces in accordance with the corresponding range of RMS threshold. The total number of success solutions is presented inside the sub-graphs in the first and fourth rows, while the $D$-value is shown inside each row. In the last column, the average RMS pattern of each success solution is depicted in the long-term time series, where the overall average RMS of success solutions by 3-, 5-, 7-, and 9-day is presented from left to right with different colours. In addition, the Sun elevation angle of $|\beta|$ is plotted on the right side of these sub-figures.

The results of SLR-only orbit determination for BDS GEO C01 is shown in Figure 6. With the increase of arc length from 3-day to 9-day, the amount of success solutions increases from 34 to 64 in the statistical results of orbit overlaps. The average RMS of 9-day solutions is $0.42,4.96$, and $2.09 \mathrm{~m}$, with the $D$-value of $93.8 \%, 89.1 \%$, and $93.8 \%$ in RTN directions respectively. However, only $22,15,10$, and 14 groups are retained for 3-, 5-, 7-, or 9-day solutions in the comparison with WUM microwave-based orbits. The reason is that many worse solutions are treated as failure solutions, which include singular solutions and eliminated solutions, and their average RMS can be tens, or even hundreds, of metres in RTN directions.

Under the premise of increasing the statistical sample, the $D$-value can improve by $20 \%-40 \%$, from $41.2 \%$ to $87.5 \%$ in $\mathrm{R}$ direction from the 3-day to 9-day solutions for comparison with WUM orbit for $\mathrm{C} 08,50 \%$ to $72.5 \%$ for $\mathrm{C} 10$, as well as $59.3 \%$ to $85.1 \%$ for C13, as seen in Figures $7-9$. The improved $D$-value indicates that the statistical sample becomes more and more stable and reliable with the increase in multi-day arc length. Meanwhile, the statistical results show that the C08's average RMS of 9-day solutions is $0.23,1.65$, and $1.71 \mathrm{~m}$ for orbit overlaps, as well as $0.29,1.97$, and $1.97 \mathrm{~m}$ for comparison with WUM orbits in RTN directions. Like C08, the average RMS of C13 is always steadily improved with the increase in the multi-day arcs, and it can reach $0.27,1.07$, and $1.44 \mathrm{~m}$, as well as 0.30 , 1.04 , and $1.40 \mathrm{~m}$ for both comparisons in RTN directions respectively. For C10, the best average RMS of orbit overlaps is statistically solved in 3-day arcs with $0.11,1.01$, and $0.90 \mathrm{~m}$ in RTN directions but only with a success rate of solutions of 5.8\% (as seen in Table 4). Indeed, its 9-day solutions have the most reliable average RMS of $0.34,1.89$, and $2.65 \mathrm{~m}$ for orbit overlaps with a success rate of $45.3 \%$, as well as $0.43,2.67$ and $3.91 \mathrm{~m}$ for comparison with WUM orbit with a success rate of $46.2 \%$ respectively. 


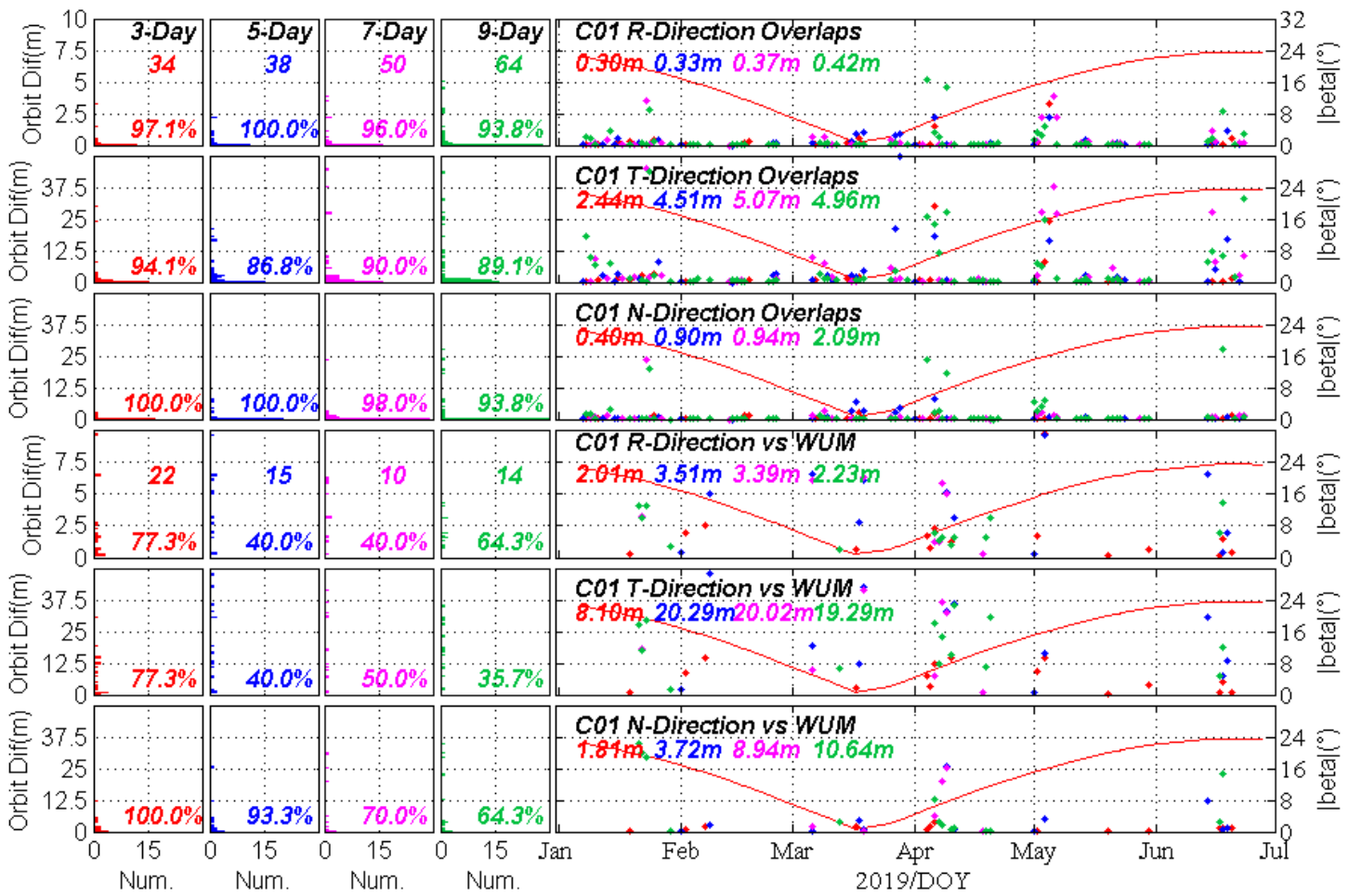

Figure 6. The results of SLR-only orbit determination for C01.

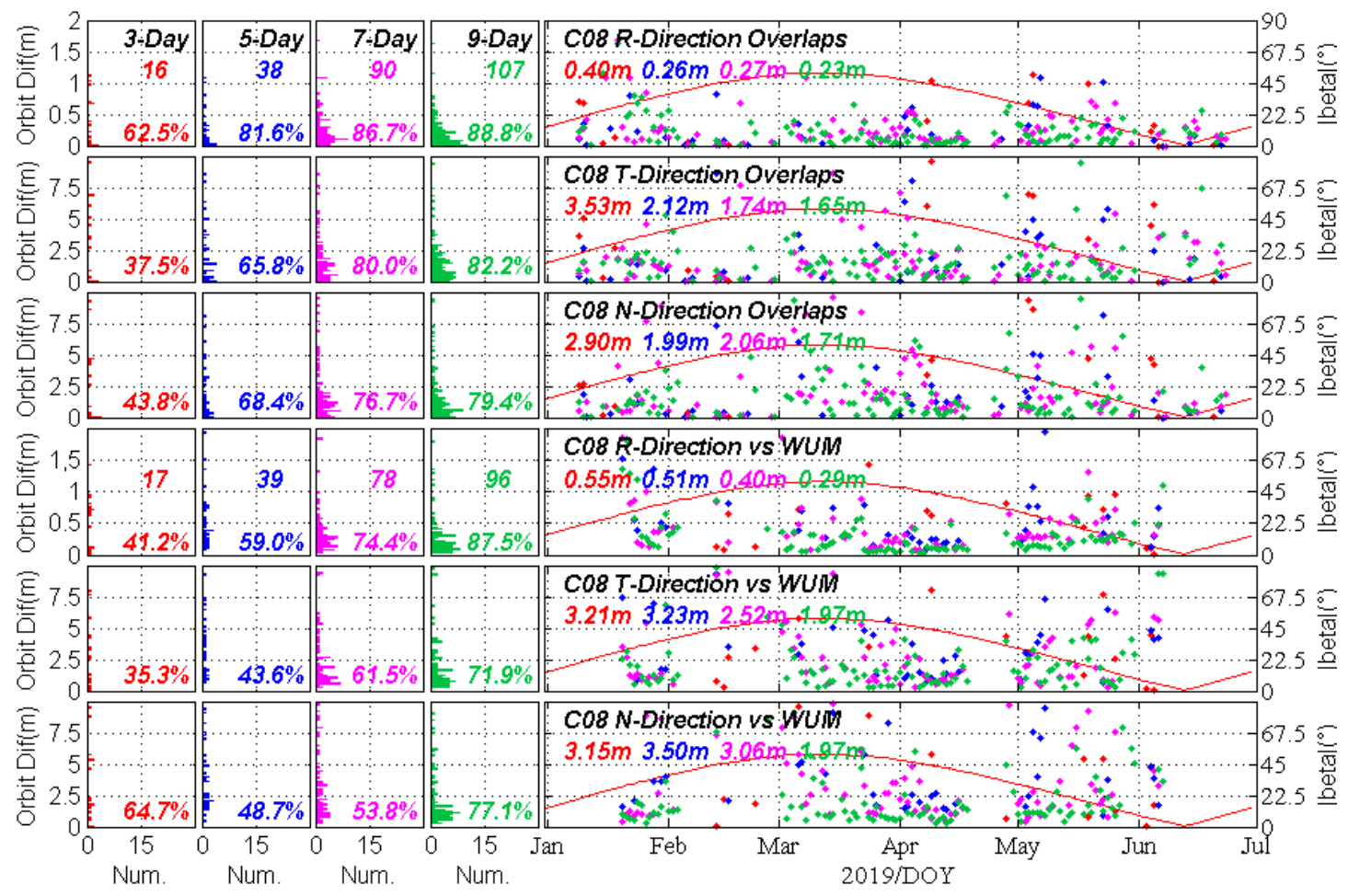

Figure 7. The results of SLR-only orbit determination for C08. 


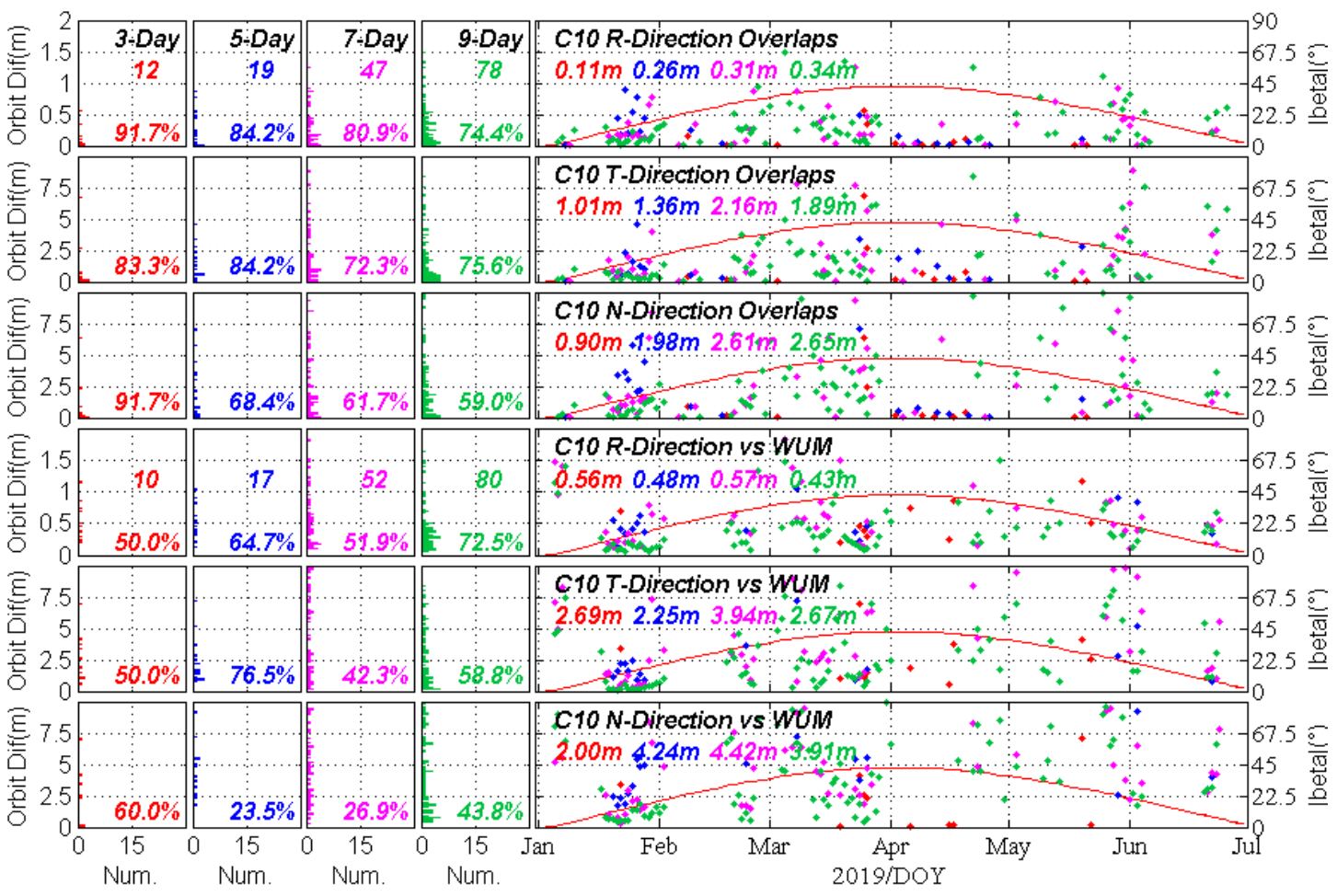

Figure 8. The results of SLR-only orbit determination for C10.

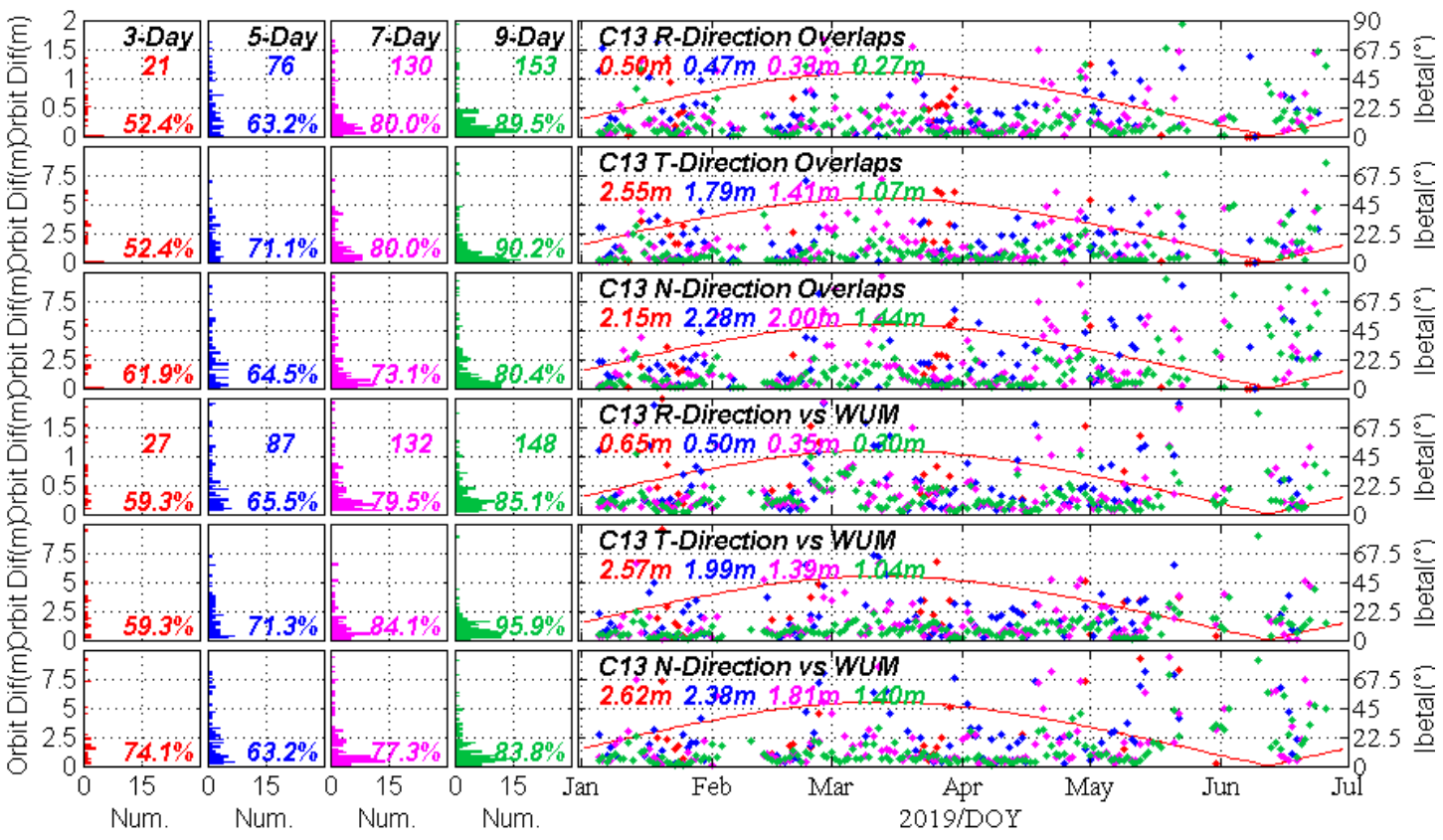

Figure 9. The results of SLR-only orbit determination for C13.

Like BDS IGSO satellites, the longer the multi-day arc, the more the success solutions. In Figure 10, no group of 9-day solutions is excluded for C11 with a $D$-value above $90 \%$ in RTN directions for both comparisons. Its average RMS is $8.5,26.0$, and $36.6 \mathrm{~cm}$ for orbit overlaps, as well as 10.1, 35.1 and 36.7 $\mathrm{cm}$ for comparison with WUM orbit respectively. The four BDS-3 satellites also have the same accuracy magnitude of SLR-only orbit determination as the BDS-2 C11, but their groups of success solutions are less than that of C11, as shown in Figures 11-14. Overall, however, comparing the average RMS of the 3-, 5-, 7-, and 9-day solutions, the accuracy of SLR-only orbit determination for the four BDS-3 
satellites can be improved from about $20 \mathrm{~cm}$ to better than $10 \mathrm{~cm}$ in $\mathrm{R}$ direction, and from sub-metre level to about $50 \mathrm{~cm}$ in both the $\mathrm{T}$ and $\mathrm{N}$ directions.

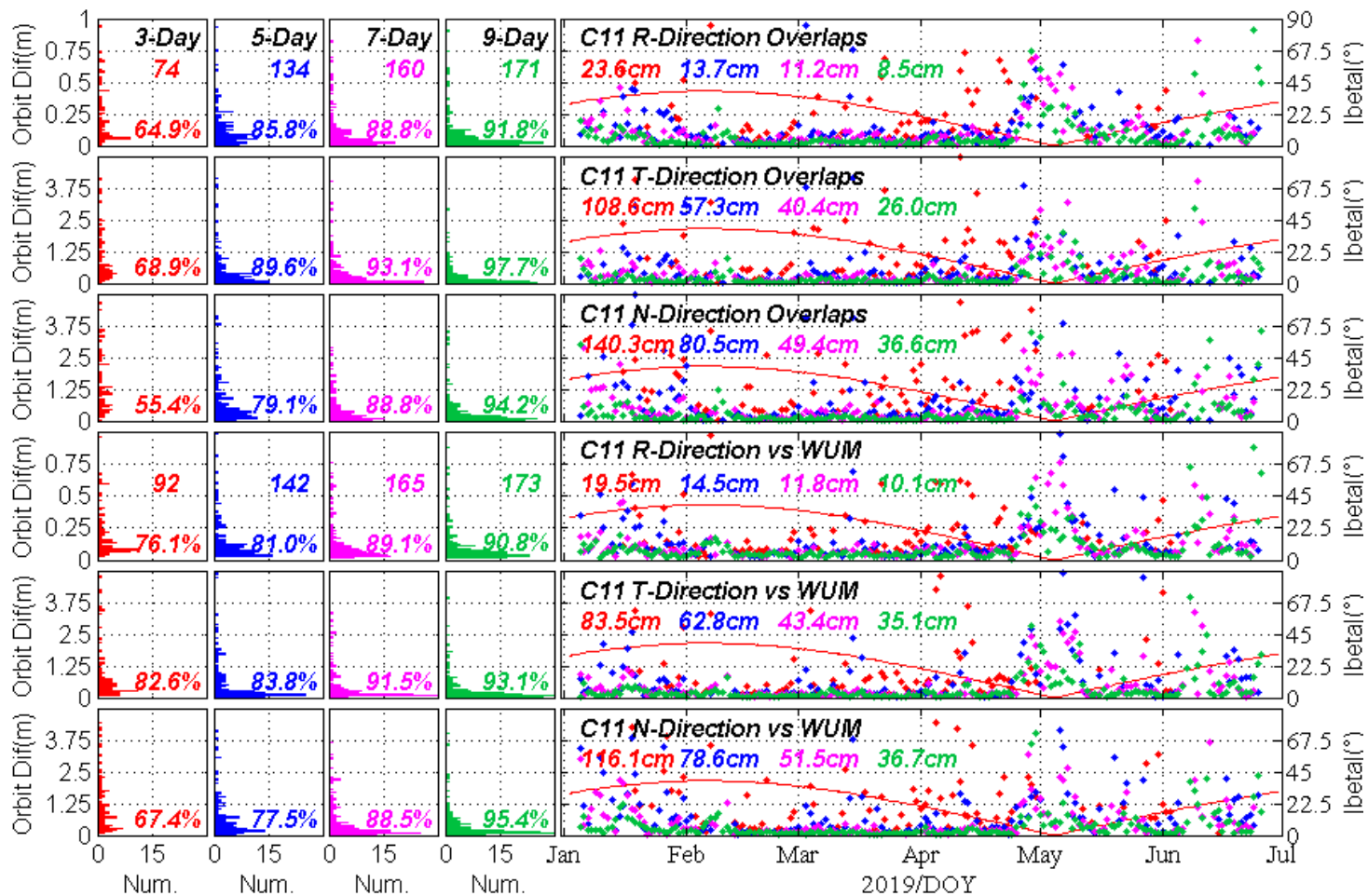

Figure 10. The results of SLR-only orbit determination for C11.

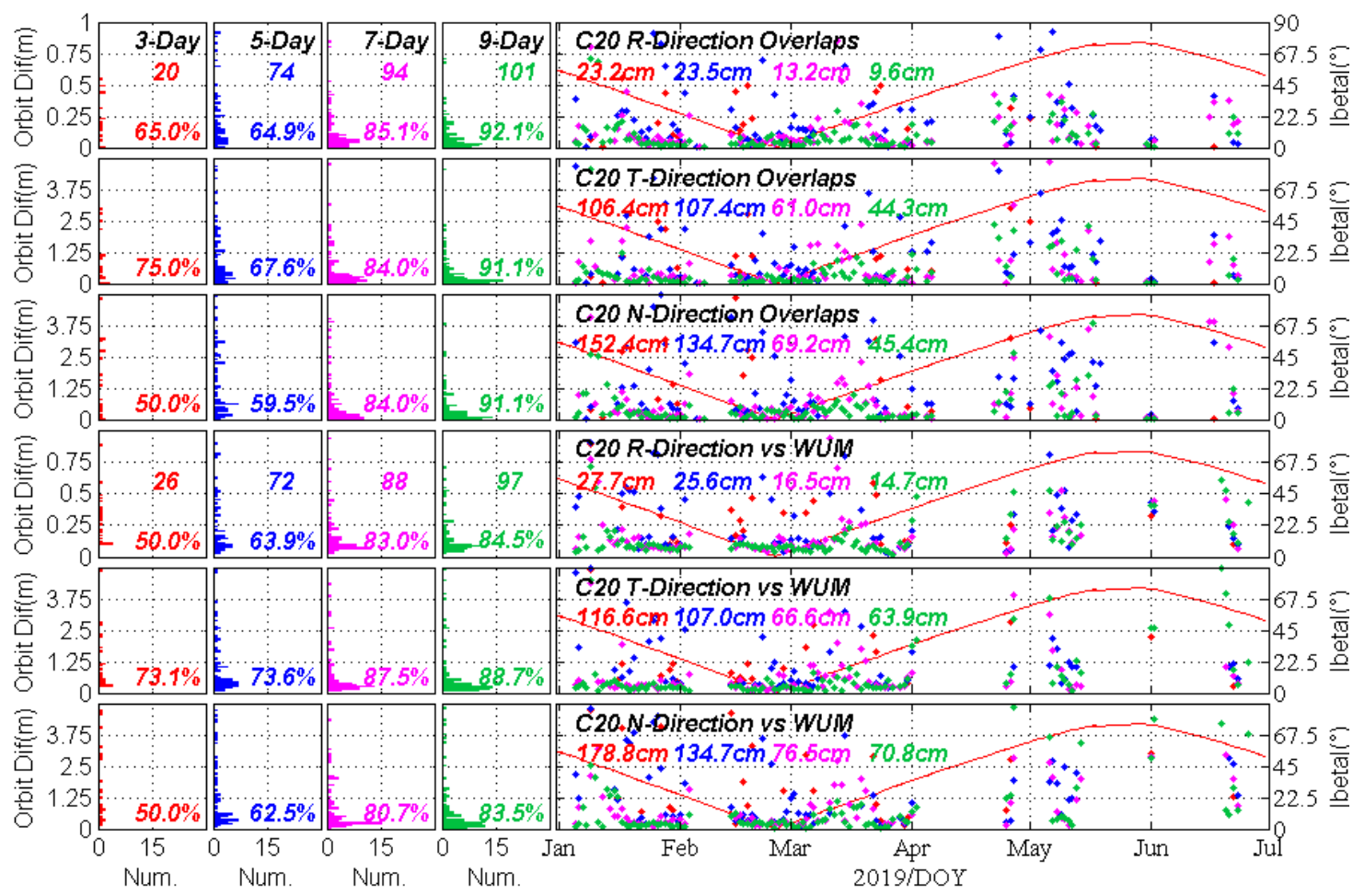

Figure 11. The results of SLR-only orbit determination for C20. 


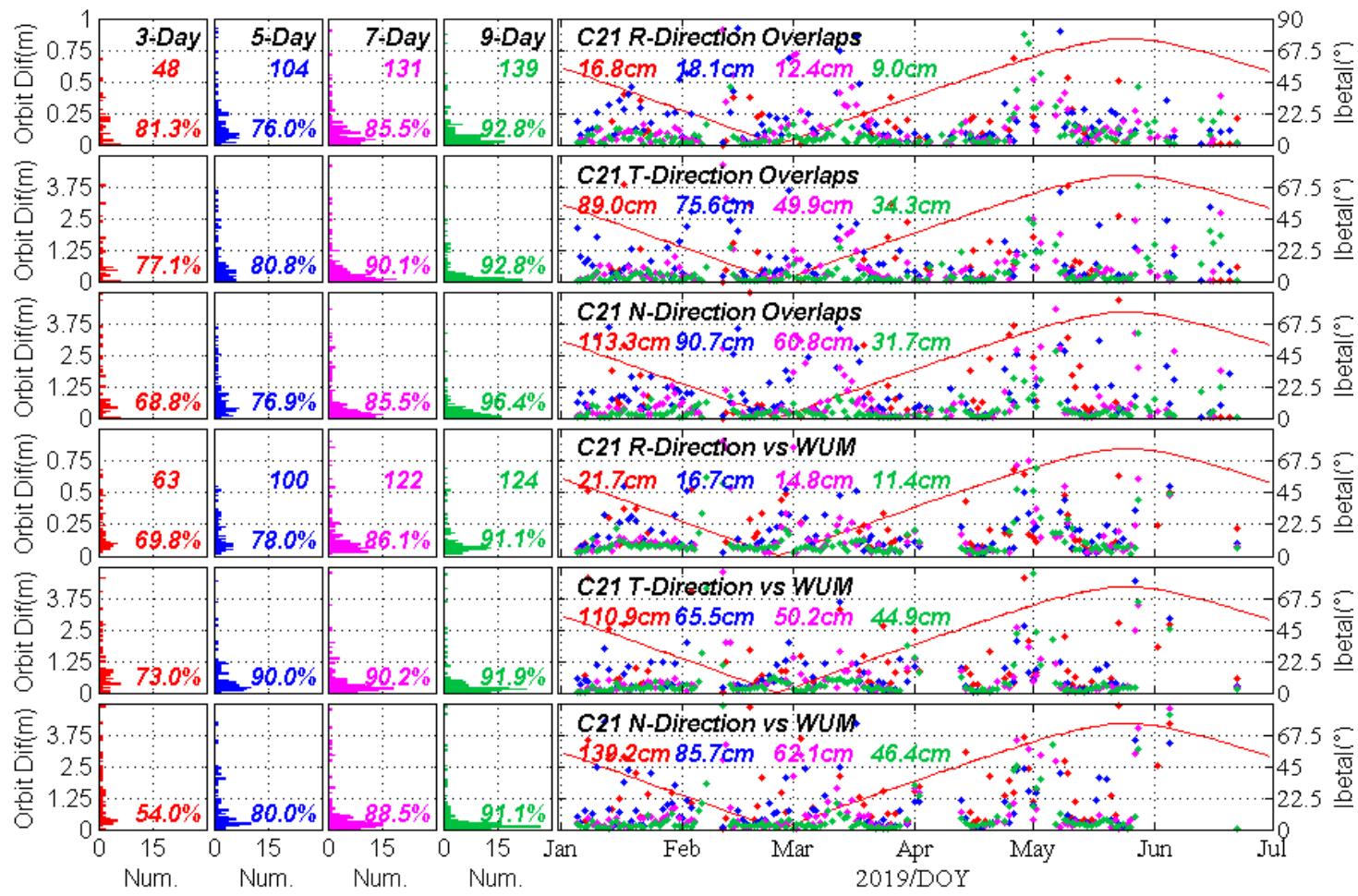

Figure 12. The results of SLR-only orbit determination for C21.

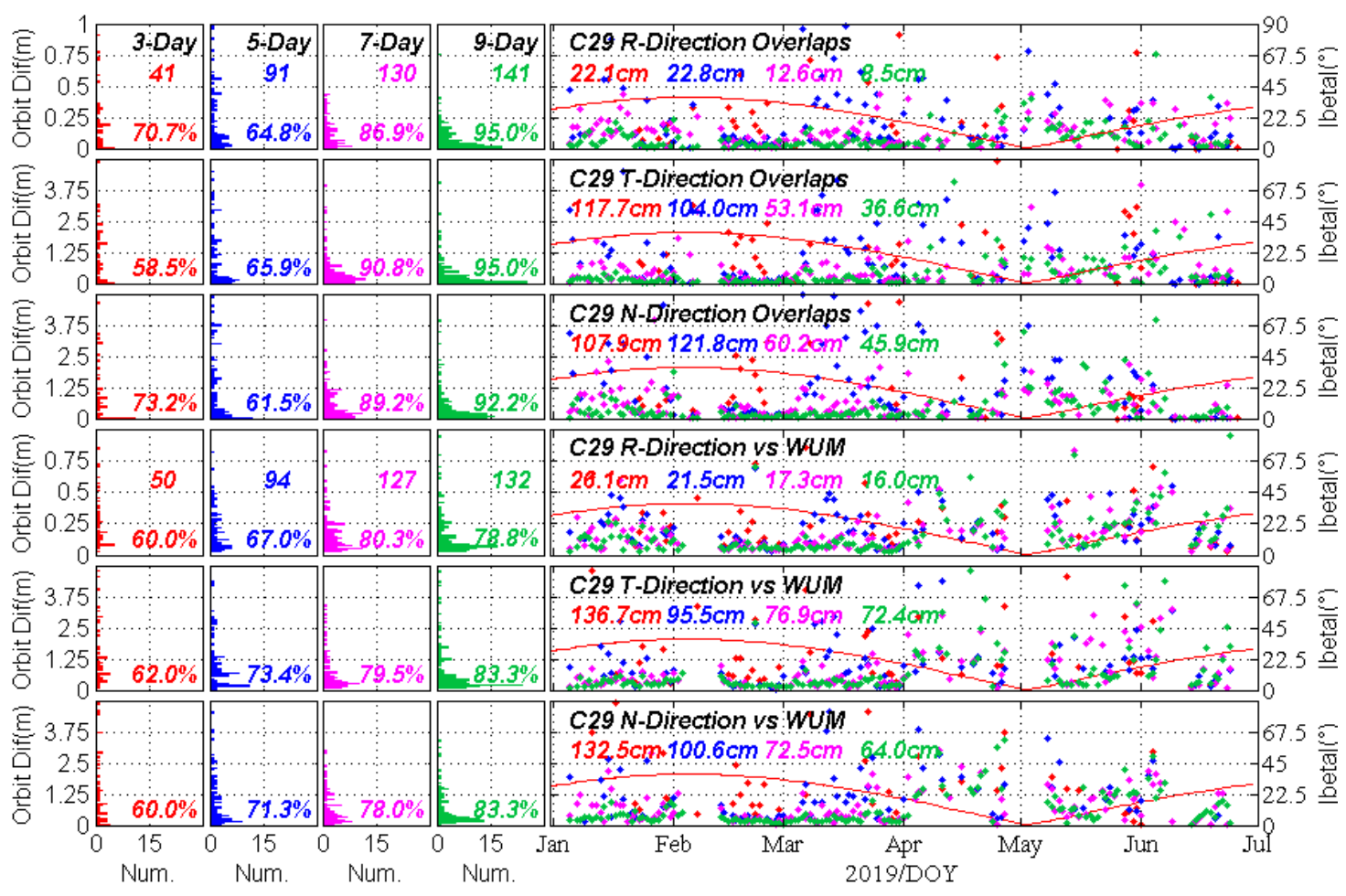

Figure 13. The results of SLR-only orbit determination for C29.

In addition, the dependency of the accuracy of the SLR-only determination on the Sun elevation angle of $|\beta|$ is found for BDS satellites, especially for BDS-2 C11. As shown in Figure 10, the RMS values of SLR-only orbit determination get larger when the $|\beta|$ is near zero for both the orbit overlaps and the comparison with WUM orbits. Because it happened during the eclipse and YM period of C11, it is probably caused by the deficiency of the SRP model and attitude model on the SLR-only orbit 
determination. While this phenomenon is not obvious for the BDS-3 C20 and C21 at present, which may be related to the attitude control modes of BDS-3, this dependency does not necessarily exist for BDS C01, C08, C10, C13, C29, and C30 at present on account of insufficient RMS values.

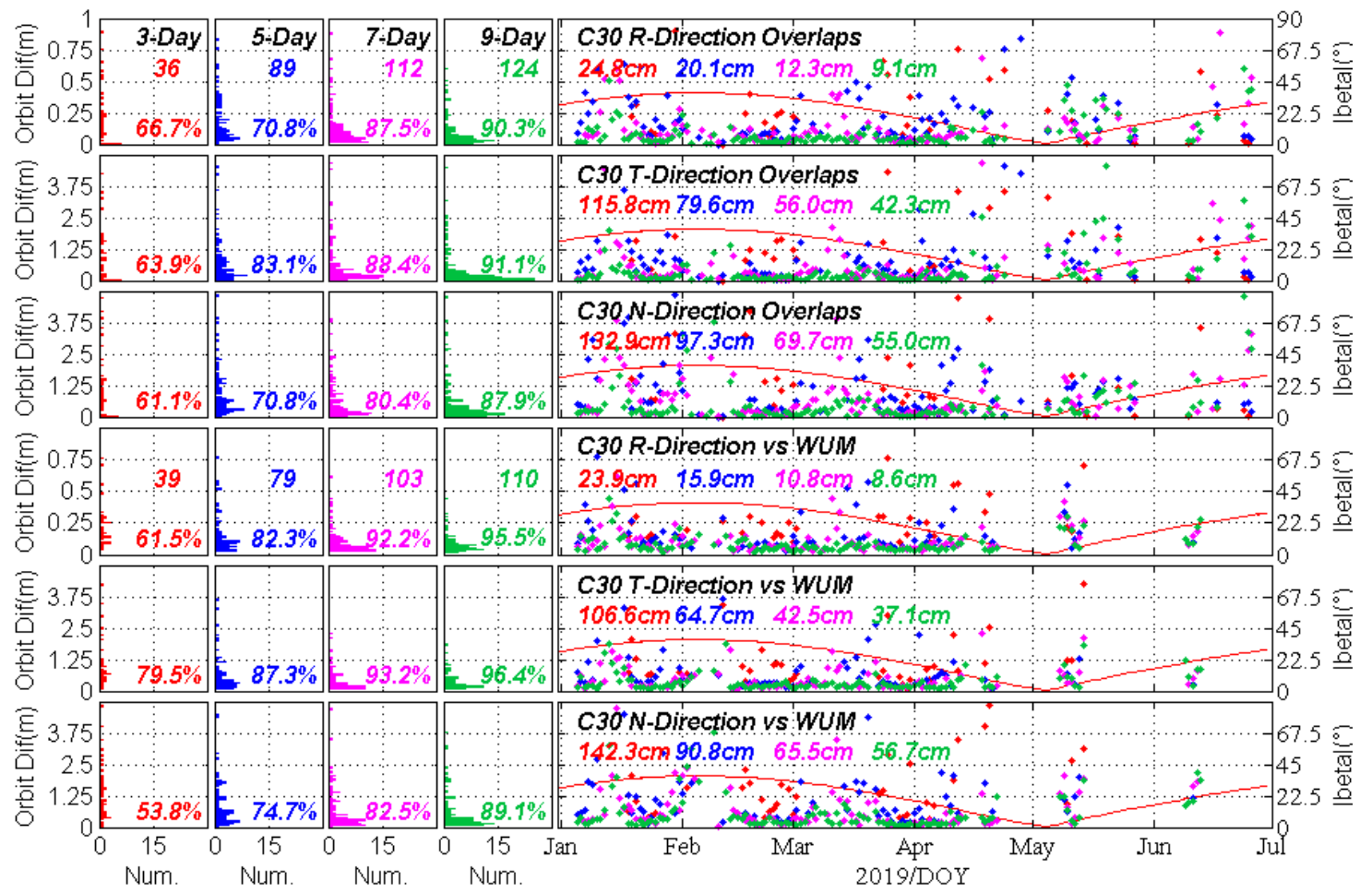

Figure 14. The results of SLR-only orbit determination for C30.

\subsection{The Overall Average 3D-RMS}

Figure 15 summarizes the overall average 3D-RMS of SLR-only orbit determination for all nine BDS satellites, where the colours blue, cyan, yellow, and red refer to the overall average 3D-RMS of 3-, 5-, 7-, and 9-day solutions. For GEO C01, the 3D-RMS of orbit overlaps is about five times better than that of the comparison with WUM orbits. The difference indicates that there must be some large systematic errors between SLR-only and microwave-based orbit determination of GEO C01 satellite, as seen in Figure 6. Interestingly, for C01, the average RMS of the 3-day arc solution is optimal among the four multi-day arc solution strategies. This is synthetically affected by the fixed orbit yaw mode of GEO, the frequent manoeuvers to adjust satellite attitude, the sparse SLR observations, and the limited visibility of SLR sites. In addition, the deterioration of the dynamic model parameters is an important factor with the lengthening of the multi-day arc solutions. Among the three IGSO satellites, the 3D-RMS of C10 are as abnormal as C01 for different multi-day arc solutions. With the increase in the arc length, the accuracy of SLR-only orbit determination improves steadily for IGSO C08 and especially for C13. The 3D-RMS of 9-day arc solutions can be significantly better than $2 \mathrm{~m}$ for C13. The regional coverage in the shape of ' 8 ' is typical characteristic of IGSO satellites, so that the observation of SLR sites are also regional because of this limitation, which is bound to reduce the quantity of the SLR observations, as well as weaken the geometric distribution of SLR sites.

Table 5 shows the maximum and minimum values of the 3D-RMS for three type of BDS satellites derived from the orbit overlaps and the comparison with WUM orbits in the half year of 2019. In all five MEO satellites, the accuracy of multi-day solutions has steadily improved from 3-day to 9-day arc solutions. The 3D-RMS of C11 is better than $1.55 \mathrm{~m}$ for 3-day arc solutions and $0.49 \mathrm{~m}$ for 9-day arc solutions. Among them, the accuracy of C11 is the best, followed by C21, C30, and C29, while that of C20 is the worst. There are three periods for which more than 10 days of SLR observations 
are missing for C20, as shown in Figure 2. When we calculate the orbit of the multi-day arc with the moving time window method, the missing data over 10 consecutive days will undoubtedly have a great impact on the SLR-only orbit determination, especially the data around the beginning and end of each interruption. Overall, the accuracy of orbit overlaps is better than that of the comparison with WUM orbits, except for the GEO C01 satellite. The BDS IGSO are characterized by the RMS values higher by a factor of about 3-5 than MEO orbits. The 9-day arc solutions are the best for BDS IGSO and MEO satellites, and their optimal 3D-RMS is $1.89 \mathrm{~m}$ and $0.49 \mathrm{~m}$ for the orbit overlaps, and $1.85 \mathrm{~m}$ and $0.55 \mathrm{~m}$ for the comparison with WUM orbits.
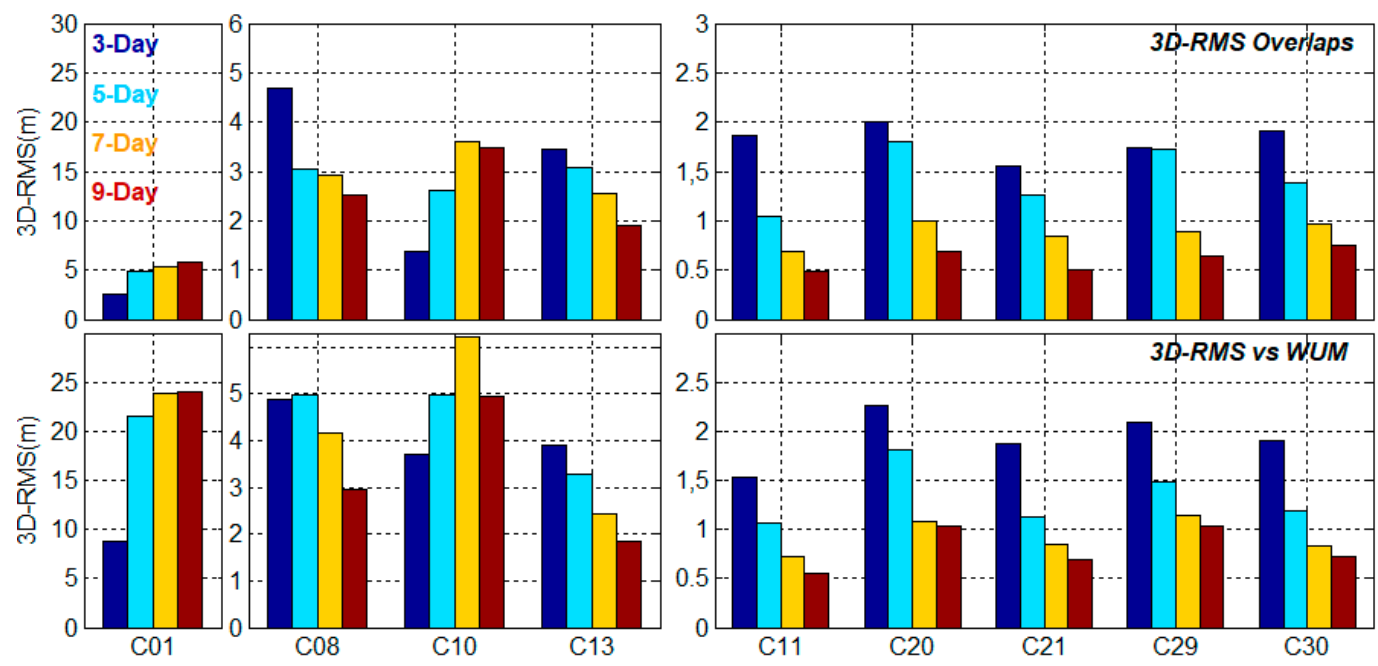

Figure 15. The overall average 3D-Root Mean Square (RMS) of SLR-only orbit determination for BDS satellites.

Table 5. The minimum and maximum values of 3D-RMS for BDS satellites (Unit: $\mathrm{m}$ ).

\begin{tabular}{|c|c|c|c|c|c|}
\hline \multicolumn{2}{|c|}{ 3D-RMS (Min/Max) } & 3-Day & 5-Day & 7-Day & 9-Day \\
\hline \multirow{3}{*}{ Overlaps } & GEO & 2.61 & 4.80 & 5.28 & 5.77 \\
\hline & IGSO & $1.38 / 4.70$ & $2.61 / 3.07$ & $2.56 / 3.60$ & $1.89 / 3.49$ \\
\hline & MEO & $1.55 / 2.00$ & $1.05 / 1.80$ & $0.69 / 0.99$ & $0.49 / 0.75$ \\
\hline \multirow{3}{*}{ Vs WUM } & GEO & 8.75 & 21.57 & 23.88 & 23.95 \\
\hline & IGSO & $3.71 / 4.89$ & $3.28 / 4.97$ & $2.42 / 6.21$ & $1.85 / 4.95$ \\
\hline & MEO & $1.54 / 2.26$ & $1.07 / 1.81$ & $0.73 / 1.14$ & $0.55 / 1.04$ \\
\hline
\end{tabular}

\subsection{The Median RMS of 9-day Solutions}

The median RMS of 9-day solutions are shown in Table 6, where the median values correspond to the direction of RTN and 3D-RMS respectively. In the statistical results of the median RMS, the accuracy of orbit overlaps is better than that of external orbit comparison for all BDS satellites. We also find that there exists a huge gap between the two modes of orbital comparisons for GEO C01, whose 3D-RMS is 100.6 and $2641.8 \mathrm{~cm}$, with a maximum difference in their T direction. For BDS-2 IGSO and MEO, these indicators are at the same accuracy level as that of results by Bury, Sośnica and Zajdel [15]. The orbit overlap 3D-RMS for IGSO C13 is $114.4 \mathrm{~cm}$ and $130.1 \mathrm{~cm}$ for comparison with WUM orbit. Overall, the 3D-RMS of BDS-2 MEO, IGSO, and GEO are in the decimetre-, metre-, and more than ten-metre levels respectively. The geometric distribution and number of the visible SLR observation sites, as well as the inaccuracy of the dynamic model, are important factors leading to this situation. Meanwhile, the median RMS of the new four BDS-3 MEO satellites are slightly lower than those of BDS-2 C11. The main reason may be influenced by the number and quality of SLR observations. The orbit overlaps median RMS of BDS-3 in R direction can reach $5 \mathrm{~cm}$, and better than $10 \mathrm{~cm}$, for comparison with the WUM orbit. 
Table 6. The median RMS of 9-day solutions (Unit: $\mathrm{cm}$ ).

\begin{tabular}{ccccccccc}
\hline \multirow{2}{*}{ PRN } & \multicolumn{4}{c}{ Overlaps } & \multicolumn{4}{c}{ Vs WUM } \\
\cline { 2 - 9 } & $\mathbf{R}$ & $\mathbf{T}$ & $\mathbf{N}$ & 3D-RMS & $\mathbf{R}$ & $\mathbf{T}$ & $\mathbf{N}$ & 3D-RMS \\
\hline C01 & 6.5 & 90.3 & 8.1 & 100.6 & 176.7 & 1796.1 & 367.5 & 2641.8 \\
C08 & 15.4 & 135.3 & 111.3 & 185.8 & 19.0 & 133.5 & 116.8 & 207.5 \\
C10 & 25.0 & 101.9 & 185.1 & 266.0 & 30.7 & 175.9 & 311.5 & 431.4 \\
C13 & 16.9 & 58.4 & 78.0 & 114.4 & 21.6 & 82.1 & 81.1 & 130.1 \\
C11 & 3.6 & 12.4 & 15.6 & 21.4 & 5.7 & 15.0 & 16.5 & 25.7 \\
C20 & 5.7 & 21.6 & 23.4 & 33.3 & 9.0 & 27.2 & 27.2 & 45.8 \\
C21 & 4.9 & 13.6 & 17.6 & 26.1 & 7.3 & 20.4 & 20.6 & 31.0 \\
C29 & 4.8 & 17.5 & 20.7 & 31.4 & 9.6 & 36.8 & 35.2 & 57.9 \\
C30 & 4.8 & 15.6 & 23.9 & 32.3 & 6.0 & 22.3 & 30.1 & 41.0 \\
\hline
\end{tabular}

\section{Discussion}

Bury, Sośnica and Zajdel [15] analysed the accuracy dependency of the SLR-only orbit determination on the number of SLR observation quantities as well as the number of SLR sites. The dependencies of 3-day arc solutions for BDS-2 MEO and IGSO satellites were involved and discussed. In the same way, we explore these dependencies of BDS-2 and BDS-3 satellites, as well as their 3-, 5-, 7-, and 9-day arc solutions in comparison with WUM orbits in this section.

\subsection{The Dependency on the Number of SLR Observations}

Figure 16 shows the dependency of RMS in RTN on the number of SLR observations. For GEO C01, the number of SLR observations can just reach 20 for the 3-day arc solutions and less than 40 for the 9-day arc solutions. For the three IGSO satellites, the maximum SLR observation number ranges from 30 to 90 for the 3-day to 9-day solutions of C08 and C10, while the number ranges from 60 to 140 for C13. Owing to its superiority of observation quantity, the SLR orbit determination accuracy of C13 is always better than that of C08 and C10 satellites under the same conditions. Similarly, the lack of observation quantity is an important factor leading to the poor accuracy of SLR-only orbit determination of C01. In five BDS MEO satellites, the number of SLR observations can be as many as 180 in the 9-day solutions of C11 satellite, followed by C21 as 140, and more than 100 for C20, C21, and C30. Overall, the dependency of median RMS on the number of SLR observations are similar for each satellite, whether it is a 3-, 5-, 7-, or 9-day solution.

The accuracy increases and improves significantly when the number of SLR observations exceeds 50 , especially in $\mathrm{T}$ and $\mathrm{N}$ directions. The dependency becomes stable and does not fluctuate greatly when the number of SLR observations reaches 80 or more. For the multi-day solution of individual satellites, the RMS of 7- or 9-day solutions are always better than that of 3- and 5-day solution when the number of SLR observations is in the range of 50 to 80, except for GEO C01. In addition, compared with the dependency of 3-day solutions RMS in RTN on the number of SLR observations for BDS-2 C08 and C11 by Bury, Sośnica and Zajdel [15], the similar dependency is reflected in Figure 16. The median RMS of 3-day solutions could still be unstable, even if the number of SLR observations reaches 80 , especially in $\mathrm{T}$ and $\mathrm{N}$ directions, though the situation becomes optimistic in the 7- and 9-day solutions for BDS satellites. It indicates that the 7- and 9-day solutions are necessary for the SLR-only orbit determination of BDS satellites under the present conditions. 

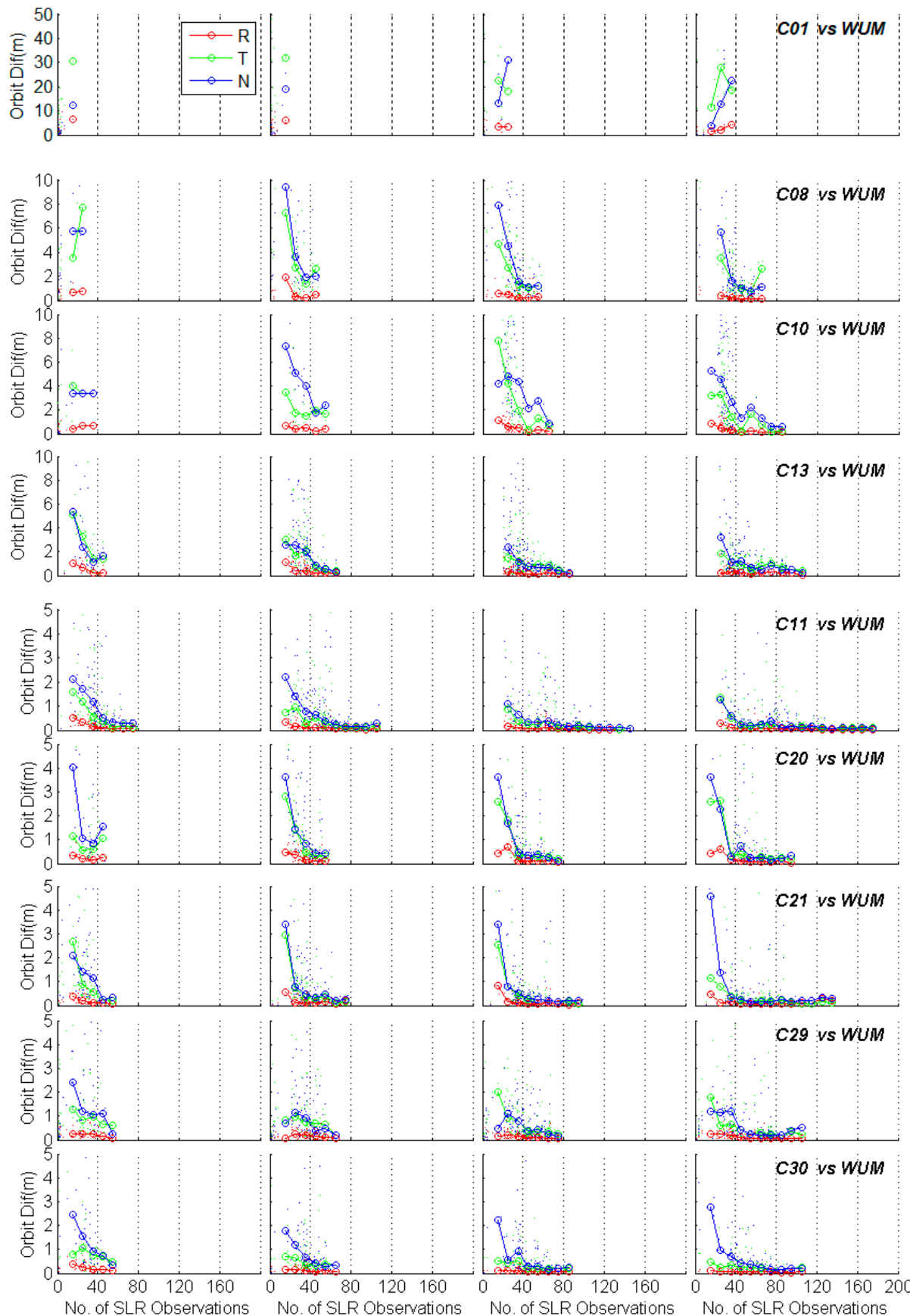

Figure 16. The dependency of median RMS on the number of SLR observations. The red, green, and blue dots, as well as dotted lines, represent values in the $\mathrm{R}, \mathrm{T}$, and $\mathrm{N}$ directions respectively. In particular, the hollow circle dotted line refers to the median RMS with a step size of 10 .

\subsection{The Dependency on the Number of SLR Sites}

The dependency of median RMS on the number of SLR sites for 3-, 5-, 7-, and 9-day solutions are displayed in Figure 17. For GEO C01, the number of SLR sites is no more than four, even in its 9-day solutions. Meanwhile, the number can reach 8 for BDS IGSO, and 12-14 for BDS MEO satellites. The influence of the SLR sites number on the accuracy of SLR orbit determinations is not negligible. Moreover, the more the number of SLR sites, the higher the accuracy of the multi-day arc solutions. When the number of SLR sites exceeds 5-6, the accuracy of multi-day arc solutions becomes more excellent and stable, especially in the $\mathrm{T}$ and $\mathrm{N}$ directions. In particular, there is no obvious regularity 
for C01, because rare success solutions are included in the multi-day arcs. Similarly, when the number of SLR sites exceeds 5, the RMS values become worse for IGSO C10 on account of fewer RMS statistics, especially in its $\mathrm{T}$ and $\mathrm{N}$ directions. In addition, the longer multi-day arc solutions have more stable RMS values because more SLR observations are included, even with the same number of SLR sites. Taking the BDS-3 MEO C21, for example, its 3-day solutions RMS becomes unstable as the number of SLR sites increases; however, the RMS values become more and more precise as the arc lengthens. In the analysis of the 3-day solution for BDS-2 C11 by Bury, Sośnica and Zajdel [15], the trend of the median RMS values is smoother than that in this contribution with the increase in the number of SLR sites. This situation may be caused by the different selection of strategies and time spans in the SLR-only orbit determination. Above all, it can be deduced that on the one hand, the longer the multi-day arcs, the greater number of SLR sites it may contain; on the other hand, the increase in the number of SLR sites strengthens the geographical distribution geometry of SLR sites, which improves the accuracy of the SLR-only orbit determination, especially in T and $\mathrm{N}$ directions.

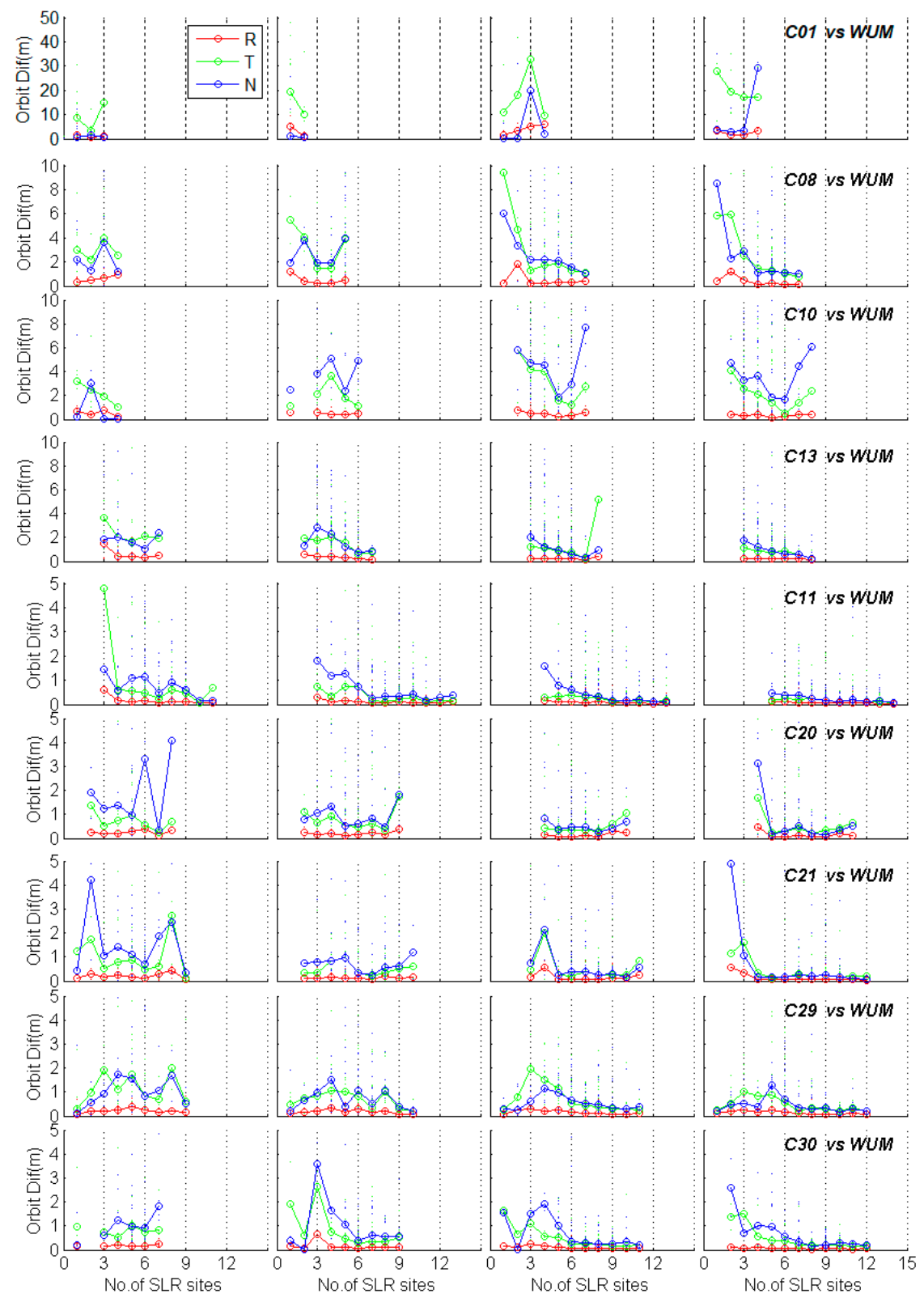

Figure 17. The dependency of median RMS on the number of SLR sites. The definition of different colour dots and circles are the same as in Figure 16. 


\section{Conclusions}

SLR is capable of making significant contributions to the orbit determination of GNSS satellites when a sufficient number of available SLR observations is provided [11], and not just for SLR validation in GNSS satellite applications. Influenced by the number of the SLR observations and the SLR sites, the SLR-only orbit determination of GNSS satellites has received less attention than that of GNSS microwave-based orbit determination. In fact, the multi-day arc solution of SLR-only orbit determination can reach a satisfactory accuracy that is comparable with that of GNSS microwave-based orbit determination. In this contribution, we determinate the SLR-only orbit of BDS-2 and BDS-3 satellites for a half-year time span since the beginning of 2019, and discuss the dependency of median RMS on the number of SLR observations and on the number of SLR sites to explore their orbit determination quality of the 3-,5-, 7-, and 9-day arc solutions.

Before SLR orbit determination, the SLR validation is performed to screen the available SLR observations. The accuracy of the microwave-based orbit of WUM is much better than that before March 2018 for BDS-2 GEO C01 [20], whose RMS of SLR residuals is $19.0 \mathrm{~cm}$, with a mean offset of $-7.5 \mathrm{~cm}$ now. The BDS-2 IGSO satellites have the credible RMS of $5.2-7.3 \mathrm{~cm}$, with the mean offset of $-4.2--1.8 \mathrm{~cm}$. Remarkably, the overall mean offset and RMS of BDS-3 MEO satellites is $-1.4-0.3 \mathrm{~cm}$ and 4.4-5.7 cm, while the RMS of the only BDS-2 MEO C11 is $3.4 \mathrm{~cm}$, with a mean offset of $0.9 \mathrm{~cm}$. As the number and geometric distribution of GNSS stations that are tracking BDS-3 signal is worse than that of BDS-2, the accuracy of BDS-3 is slightly worse than that of BDS-2.

With only a slight difference in SLR observations data preprocessing strategies, BDS GEO C01 is processed synchronously with BDS IGSO and MEO satellites for SLR-only orbit determination. Unfortunately, the SLR-only orbit determination accuracy of C01 is not very promising on account of the extremely rare SLR observations. Overall, the SLR-only orbit determination accuracy of BDS-2 GEO satellite only can reach a level of 10 metres or worse while the 9-day arc solutions present the best orbit accuracy in our multi-day SLR-only orbit determination for BDS IGSO and MEO satellites. Although the SLR-only orbit determination accuracy of BDS-3 MEO satellites are slightly worse than BDS-2 MEO C11 under the same number of SLR sites and SLR observations, the SLR-only orbit determination accuracies of all BDS MEO are on the same level. The 9-day overlaps median RMS of BDS MEO in RTN directions are evaluated at 3.6-5.7, 12.4-21.6, and 15.6-23.9 cm respectively, as well as 5.7-9.6, 15.0-36.8, and 16.5-35.2 cm for the comparison with WUM precise orbits, while these values of BDS IGSO are larger by a factor of about 3-10 than BDS MEO orbits in their corresponding RTN directions. Furthermore, the optimal average 3D-RMS of 9-day overlaps is 0.49 and $1.89 \mathrm{~m}$ for BDS MEO and IGSO respectively, as well as 0.55 and $1.85 \mathrm{~m}$ in comparison with WUM orbits. Besides, the SLR-only orbit determination accuracy of IGSO C13 is better than that of IGSO C08 and C10, since it has more available SLR observations. However, limited by the geographical distribution and number of the available SLR sites, the SLR-only orbit determination accuracy of IGSO is worse than that of MEO, even with the same SLR observations.

In summary, SLR is the space geodetic technique with the highest single independent ranging and absolute positioning accuracy relative to the centre of the Earth. As things stand, the SLR-only orbit determination accuracy of BDS GEO is more than tens of metres while that of BDS IGSO and MEO is at the level of submetre and decimetre. Though the SLR-only orbit determination of GNSS satellites suffers from some limitations, it will play an important and positive role in scientific research that does not require so high an orbital accuracy, such as the orbit determination and prediction of space debris. Also, the combined orbit determination using the space geodesy technologies of GNSS, SLR, as well as VLBI and DORIS, are bound to be the development trends of space technology in the future.

Author Contributions: Conceptualization, T.X.; Formal analysis, H.Y.; Funding acquisition, T.X.; Methodology, H.Y.; Project administration, T.X.; Software, H.Y.; Validation, W.N., F.G. and M.G.; Writing - original draft, H.Y. and W.N.; Writing - review \& editing, T.X. 
Funding: This study is financially supported by the National Natural Science Foundation of China (Grant No. 41874032, 41731069 and 41574013) and the National Key Research \& Development Program of China (2016YFB0501701).

Acknowledgments: Thanks are due to ILRS, CDDIS, EDC, IGS ACs, and WUM for providing the SLR data and orbits of BeiDou Navigation Satellite System. The authors are thankful to Dr Adrià Rovira Garcia, from group of Astronomy and Geomatics (gAGE), Universitat Politecnica de Catalunya (UPC) at Barcelona, Spain, who revised the manuscript. Finally, the authors are also grateful for the comments and remarks of three reviewers, which helped improve the manuscript significantly.

Conflicts of Interest: The authors declare no conflict of interest.

\section{References}

1. Yang, Y.; Xu, Y.; Li, J.; Yang, C. Progress and performance evaluation of BeiDou global navigation satellite system: Data analysis based on BDS-3 demonstration system. Sci. China Earth Sci. 2018, 61, 614-624. [CrossRef]

2. Yang, Y.; Gao, W.; Guo, S.; Mao, Y.; Yang, Y. Introduction to BeiDou-3 navigation satellite system. Navigation 2019, 66. [CrossRef]

3. Degnan, J.J.; Pavlis, E.C. Laser Ranging to GPS Satellites with Centimeter Accuracy. GPS World 1994, 1994, 62-70.

4. Pavlis, E.C. Comparison of GPS S/C orbits determined from GPS and SLR tracking data. Adv. Space Res. 1995, 16, 55-58. [CrossRef]

5. Schutz, B.E. Synergism of SLR and GPS. In Proceedings of the Satellite Laser Ranging in the 1990s: Report of the 1994 Belmont Workshop, Elkridge, MD, USA, 1-2 February 1994.

6. Zhu, S.Y.; Reigber, C.; Kang, Z. Apropos laser tracking to GPS satellites. J. Geod. 1997, 71, 423-431. [CrossRef]

7. $\quad$ Eanes, R.J.; Nerem, R.S.; Abusali, P.A.M.; Bamford, W.; Ries, J.C. GLONASS Orbit Determination at the Center for Space Research; University of Texas at Austin: Austin, TX, USA, 1999.

8. Melachroinos, S.A.; Perosanz, F.; Deleflie, F.; Biancalel, R.; Laurain, O.; Exertier, P. GIOVE-A and GPS-35/36 orbit determination and analysis of dynamical properties based on SLR-only tracking data. In Proceedings of the 15th International Workshop on Laser Ranging, Canberra, Australia, 15-20 October 2006.

9. Schonemann, E.; Springer, T.; Otten, M.; Becker, M.; Dow, J. GIOVE-A precise orbit determination from microwave and satellite laser ranging data-first perspectives for the Galileo constellation and its scientific use. In Proceedings of the First Colloquium on Scientific and Fundamental Aspects of the Galileo Programme, Toulouse, France, 1-4 October 2007; Available online: http://ilrs.gsfc.nasa.gov/docs/2007_Schoenemann_ Toulouse_paper.pdf (accessed on 19 October 2019).

10. Urschl, C.; Beutler, G.; Gurtner, W.; Hugentober, U.; Ploner, M. Orbit determination for GIOVE-A using SLR tracking data. In Proceedings of the 15th International Workshop on Laser Ranging, Canberra, Australia, 15-20 October 2006; pp. 40-46.

11. Flohrer, C. Mutual Validation of Satellite-Geodetic Techniques and its Impact on GNSS Orbit Modeling; Geodätisch-geophysikalische Arbeiten in der Schweiz; Swiss Geodetic Commission: Zürich, Switzerland, 2008; Volume 75, ISBN 978-3-908440-19-2.

12. Zhao, G.; Zhou, S.S.; Zhou, X.H.; Wu, B. Precise Orbit Determination of BeiDou Satellites Using Satellite Laser Ranging; Springer: Berlin/Heidelberg, Germany, 2013; pp. 221-229.

13. Zhao, G.; Zhou, S.S.; Zhou, X.H.; Wu, B. Comparison on orbit precisions of different types of navigation satellites based on SLR tracking data. In Proceedings of the 18th International Workshop on Laser Ranging, Goar, Germany, 1-3 June 2013.

14. Bury, G.; Sośnica, K.; Zajdel, R. How many SLR observations and how many stations are needed for deriving high-quality multi-GNSS orbits? In Proceedings of the 2017 ILRS Technical Workshop, Riga, Latvia, 2-5 October 2017.

15. Bury, G.; Sośnica, K.; Zajdel, R. Multi-GNSS orbit determination using satellite laser ranging. J. Geod. 2019, 1-17. [CrossRef]

16. Beutler, G.; Brockmann, E.; Gurtner, W. Extended orbit modeling techniques at the CODE processing center of the International GPS Service for geodynamics (IGS): Theory and initial results. Manuscr. Geod. 1994, 19, 367-386. 
17. Standish, E.M., Jr. The observational basis for JPL's DE 200, the planetary ephemerides of the Astronomical Almanac. Astron. Astrophys. 1990, 233, 252-271.

18. Petit, G.; Luzum, B. IERS Conventions. (IERS Technical Note; No. 36) Frankfurt am Main: Verlag des Bundesamts für Kartographie und Geodäsie; IERS Technical Note: Frankfurt, Germany, 2010; p. 179.

19. Mendes, V.B.; Pavlis, E.C. High-accuracy zenith delay prediction at optical wavelengths. Geophys. Res. Lett. 2004, 31, 189-207. [CrossRef]

20. Yang, H.; Xu, T.; Nie, W.; Gao, F.; Guan, M. SLR validation and evaluation on BDS precise orbits from 2013 to 2018. Adv. Space Res. 2019, 64, 475-490. [CrossRef]

21. Dach, R.; Lutz, S.; Walser, P.; Fridez, P. Bernese GNSS Software Version 5.2; University of Bern, Bern Open Publishing: Bern, Switzerland, 2015.

22. Beutler, G.; Brockmann, E.; Hugentobler, U.; Mervart, L.; Rothacher, M.; Weber, R. Combining consecutive short arcs into long arcs for precise and efficient GPS Orbit Determination. J. Geod. 1996, 70, 287-299. [CrossRef]

23. Lutz, S.; Meindl, M.; Steigenberger, P.; Beutler, G.; Sośnica, K.; Schaer, S.; Dach, R.; Arnold, D.; Thaller, D.; Jäggi, A. Impact of the arc length on GNSS analysis results. J. Geod. 2016, 90, 365-378. [CrossRef]

24. Prange, L.; Orliac, E.; Dach, R.; Arnold, D.; Beutler, G.; Schaer, S.; Jäggi, A. CODE's five-system orbit and clock solution-The challenges of multi-GNSS data analysis. J. Geod. 2017, 91, 1-16. [CrossRef]

25. Urschl, C.; Gurtner, W.; Hugentobler, U.; Schaer, S.; Beutler, G. Validation of GNSS orbits using SLR observations. Adv. Space Res. 2005, 36, 412-417. [CrossRef]

26. Urschl, C.; Beutler, G.; Gurtner, W.; Hugentobler, U.; Schaer, S. Contribution of SLR tracking data to GNSS orbit determination. Adv. Space Res. 2007, 39, 1515-1523. [CrossRef]

27. Sośnica, K.; Thaller, D.; Dach, R.; Steigenberger, P.; Beutler, G.; Arnold, D.; Jäggi, A. Satellite laser ranging to GPS and GLONASS. J Geod. 2015, 89, 725-743. [CrossRef]

28. Yang, H.; Xu, T.; Sun, D. Validation of GPS36 Satellite CODE Precise Orbit with SLR Measurements; China Satellite Navigation Conference (CSNC) 2016 Proceedings: Volume III, Lecture Notes in Electrical Engineering 390; Springer: Singapore, 2016. [CrossRef]

29. Sośnica, K.; Prange, L.; Kaźmierski, K.; Bury, G.; Drożdżewski, M.; Zajdel, R.; Hadas, T. Validation of Galileo orbits using SLR with a focus on satellites launched into incorrect orbital planes. J. Geod. 2018, 92, 131-148. [CrossRef]

30. Zajdel, R.; Sośnica, K.; Bury, G. A New Online Service for the Validation of Multi-GNSS Orbits Using SLR. Remote Sens. 2017, 9, 1049. [CrossRef]

31. Zhao, Q.; Wang, C.; Guo, J.; Wang, B.; Liu, J. Precise orbit and clock determination for BeiDou-3 experimental satellites with yaw attitude analysis. GPS Solut. 2017, 22, 4. [CrossRef]

32. Wang, C.; Guo, J.; Zhao, Q.; Liu, J. Yaw attitude modeling for BeiDou I06 and BeiDou-3 satellites. GPS Solut. 2018, 22, 117. [CrossRef]

(C) 2019 by the authors. Licensee MDPI, Basel, Switzerland. This article is an open access article distributed under the terms and conditions of the Creative Commons Attribution (CC BY) license (http://creativecommons.org/licenses/by/4.0/). 